\title{
Narrative Bewältigung von einschneidenden Erlebnissen eines Rückkehrers in der deutschen und türkischen Lebenswelt
}

\begin{abstract}
Mein Beitrag entstand im Rahmen meiner biografie- und interaktionsanalytischen Studie zu sozialen und sprachlichen Erfahrungen junger „Rückkehrer/innen“, d. h. junger Frauen und Männer türkischer Herkunft, die in Deutschland oder Österreich aufwuchsen, und als Jugendliche bzw. junge Erwachsene in die Türkei migrierten. Furkan, der Informant, den ich hier vorstelle, schildert Ausgrenzungserfahrungen in Deutschland aufgrund seiner ethnischen Herkunft und Anpassungsprobleme in der Türkei aufgrund sprachlicher und sozialer Auffälligkeiten.

Ziel meiner Analyse ist es, die verschiedenen Phasen seiner Lebensgeschichte in beiden Lebenswelten zu beschreiben, den Zusammenhang zwischen Ausgrenzungserlebnissen, ihrer Deutung und ihrer narrativen Bewältigung zu rekonstruieren und die Unterschiede zwischen der Schilderung in beiden Lebenswelten herauszuarbeiten. Auf dieser Basis lässt sich die narrative Bewältigung der Erlebnisse in Kindheit und früher Jugend in Deutschland mit Erzählformen für Traumata in Beziehung setzen.

This article is based on data from my biographically and interactionally oriented pilot study on the sociolinguistic experiences of "returners", i. e. young men and women of Turkish origin who grew up in Germany or Austria and who migrated to Turkey as young adults. Furkan, the informant who figures here, describes experiences of exclusion in Germany because of his ethnic background, and problems in adjusting to life in Turkey because of his social and linguistic conspicuousness.

The aim of my analysis is to describe the different phases of his biography in the two different environments, to reconstruct the relationship between experiences of exclusion in both environments, the ways they are interpreted and presented in his narrative, and finally to analyse the differences of the narratives referring to one or the other environment. On the basis of all of this, Furkan's narratives about his experiences as a child in Germany may be related to narrative forms characteristic of coping with traumatic experiences.
\end{abstract}

\section{Einleitung: Gegenstand und Ziel des Beitrags ${ }^{1}$}

In meiner biografie- und interaktionsanalytischen Pilotstudie „Deutsch-Türkische Migration: Die Darstellung narrativer Identitäten von Studentinnen in Istanbul“ (Devran 2017) untersuchte ich die sozialen und sprachlichen Erfahrungen von drei jungen Frauen, die in Deutschland und Österreich aufwuchsen, und als Jugendliche bzw. junge Erwachsene in die Türkei migrierten. Typische Erfahrungen der „Rückkehrerinnen“2 sind beispielsweise

1 Der Beitrag ist während eines dreimonatigen Forschungsaufenthalts am Leibniz-Institut für Deutsche Sprache (IDS) Mannheim im Sommer 2018 entstanden. Mein besonderer Dank gilt dem Deutschen Akademischen Austauschdienst für seine großzügige finanzielle Förderung durch ein DAAD Stipendium für ausländische Hochschullehrer.

2 Der Begriff „Rückkehrer/innen“ ist in Anführungszeichen gesetzt, da es sich nicht um Rückkehrer/innen im eigentlichen Sinne handelt. Die betreffenden Personen haben die Bezeichnung von ihren Eltern bzw. Großeltern übernommen, die als Arbeitermigrant/innen nach Deutschland migriert und später in die Türkei zurückgekehrt sind (vgl. Devran 2017, S. 14). 
die Vorurteile gegenüber Türken in Schule und Gesellschaft in Deutschland. In der Türkei werden die Informantinnen aufgrund sprachlicher und sozialer Auffälligkeiten ausgegrenzt; sie fühlen sich als „Almanc1“ abgelehnt und abgewertet. Aufgrund von sprachlichen und sozio-kulturellen Gemeinsamkeiten fühlen sie sich in gleichaltrigen „Rückkehrergruppen" am wohlsten; dort gelingt ihnen die soziale Verortung als Deutsch-Türken oder Weltbürger (ebd., S. 17 ff.).

Furkan, der Informant, den ich im Folgenden vorstelle, schildert ebenfalls das Erleben ethnischer Ausgrenzung in der Schule in Deutschland, ebenso wie das Erleben sprachlicher und kultureller Anpassungsprobleme in der Türkei. Auch ihm gelingt es erst in der deutsch-türkischen „Rückkehrergruppe“ in der Türkei ein Gefühl von Zugehörigeit und ehrgeizige berufliche Perspektiven zu entwickeln. Ähnliche Erfahrungen schildert auch Mehmet Daimagüler (2011) in seiner Biografie. Er ist der Sohn einer Gastarbeiterfamilie, erfährt als Grundschüler die Selektion in die Sonderschule, erlebt Diskriminierung im sozialen Umfeld in Deutschland und schwankt zwischen zwei Lebenswelten. Später wird er Absolvent von Harvard und Yale, praktiziert als Anwalt und ist „Young Leader ${ }^{\text {"63 }}$ der Atlantik-Brücke ${ }^{4}$ und „Global Young Leader“"5.

Interessant an Furkans Erzählungen ist, dass er die z.T. dramatischen Erfahrungen in Deutschland anders darstellt als seine Erfahrungen in der Türkei. Die Erinnerungen an die deutsche Lebenswelt sind geprägt von Gewalt und Ausgrenzung, die er als traumatisches Erleben schildert, während seine Erfahrungen in der Tükei eher als Anpassungsprobleme, als schmerzhaftes Eingewöhnen in eine fremde Lebenswelt geschildert werden.

Ziel meiner Analyse ist es, die verschiedenen Phasen von Furkans Lebensgeschichte in beiden Lebenswelten zu beschreiben, den Zusammenhang zwischen Ausgrenzungserfahrungen, ihrer Deutung und ihrer narrativen Bewältigung zu rekonstruieren und die Unterschiede zwischen der Schilderung in beiden Lebenswelten herauszuarbeiten. Auf dieser Basis lässt sich die narrative Bewältigung der Erlebnisse in Kindheit und früher Jugend in Deutschland mit Erzählformen für Traumata in Beziehung setzen.

3 Das „World Economic Forum“ in Davos kürte Mehmet Gürcan Daimagüler im Jahr 2005 auf Initiative des damaligen Bundeskanzlers Gerhard Schröder zum „,Young Global Leader“. Er ist bis heute Mitglied dieser Organisation.

4 Die Atlantik-Brücke wurde im Jahr 1952 in der BRD gegründet. Ziel ist es, die persönliche Begegnung zwischen deutschen und amerikanischen Führungskräften zu fördern. Darüber hinaus bietet sie verschiedenen Entscheidungsträgern aus Wirtschaft, Politik und Wissenschaft von beiden Seiten des Atlantiks eine Plattform für Treffen und für die globale Vernetzung von Akteuren. Heute beschäftigt sich die Atlantik-Brücke unter anderem neben außenpolitischen Themen auch mit Fragen der Digitalisierung, Klima und Energie. (Online: www.atlantik-bruecke.org, Stand: 23.6.2019).

5 Young Global Leader ist eine Community mit über 1000 Mitgliedern aus verschiedenen Berufsgruppen, die vom World Economic Forum (WEF) unterstützt wird. Ziel des WEF ist es institutionalisierte Prozesse zu etablieren und somit Plattformen für kontinuierliche Aktivitäten und Interaktionen aufzustellen. Im Fokus steht dabei die öffentlich-private Zusammenarbeit von jungen globalen Führungskräften anzuregen, einen intensiven Austausch und gute Beziehungen zwischen den Kulturen zu pflegen und eine innovative Form der Zusammenarbeit zur Bewältigung drängender lokaler und globaler Herausforderungen zu fördern. (Online: www.younggloballeaders.org, Stand: 14.6.2019). 


\section{Theoretische und methodische Grundlagen: Positionierungskonzept, Agentivität und Traumaanalyse}

Beim vorliegenden Beitrag handelt es sich um eine qualitative Untersuchung, die an biografische und interaktionistische Ansätze anknüpft. Datengrundlage ist eine Lebensgeschichte, dargestellt aus der Retrospektive, in der es um Erfahrungen in Bezug auf die erlebte Migration geht. ${ }^{6}$

Der biografische Ansatz bezieht sich auf die Rekonstruktion der mit der Veränderung der Lebenswelten einhergehenden Entwicklungen, deren Sinnkonstruktionen und die in den individuellen Darstellungen durchscheinenden übergreifenden gesellschaftlichen Strukturen. Das heißt, in den rekonstruierten Handlungsdarstellungen des Informanten sind zugleich die für die Familie, die Gruppe und in der jeweiligen sozialen Lebenswelt geltenden Überzeugungen, Werte und Normen mitenthalten. Von Interesse ist dabei, wie die sozialen und sprachlichen Erfahrungen in den verschiedenen Lebenswelten aus der Rückschau dargestellt, interpretiert und bewertet werden (vgl. Keim et al. 2012, S. 30, 32).

Der interaktionistische Ansatz knüpft an ethnomethodologische, ethnografische und konversationsanalytische Ansätze an. Er geht von der lokalen Hervorbringung von Identitätsaspekten aus, wie sie in Gesprächssequenzen emergieren und in Verbindung zu Bedeutungsrahmen gebracht werden, auf die die Beteiligten bei der Aushandlung rekurrieren. $^{7}$

\subsection{Positionierungskonzept und Agentivität}

Für die Analyse des Gesprächs beziehe ich mich auf die in der Erzählforschung entwickelten Konzepte, die die Darstellung narrativer Identität zu erfassen suchen, vor allem auf das Positionierungskonzept, das Lucius-Hoene/Deppermann (2004) und Deppermann (2013) als Instrument zur empirischen Erforschung narrativer Identitäten auf der Basis von autobiografischen Erzählungen vorschlagen. Die Autoren argumentieren, dass mithilfe des Positionierungskonzepts ein materialgestützter Zugang zu Prozessen der Identitätskonstitution in Erzählungen geleistet werden kann, ,da es die identitätsrelevanten darstellerischen wie performativen Handlungen von Erzählern zu rekonstruieren erlaubt" (LuciusHoene/Deppermann 2004, S. 166). Dabei wird zwischen verschiedenen Ebenen und

\footnotetext{
Das biografisch-narrative Interview, eingeführt von Fritz Schütze (1983) und von Gabriele Rosenthal (1995) weiterentwickelt, hat sich als Erhebungsverfahren in der qualitativen Sozialforschung etabliert. Die Durchführung des Interviews erfolgt in drei Phasen. Die erste Phase wird eingeleitet durch die Erzählaufforderung der Interviewerin, ausführlich über Erlebnisse und Erfahrungen in den verschiedenen biografischen Phasen zu erzählen. Die möglichst zurückhaltende, interessierte und empathische Gesprächsführung der Interviewerin hat dabei eine unterstützende und motivierende Funktion. In der zweiten Phase folgen dann die auf die Erzählung generierenden Fragen oder Bemerkungen. Erst in der letzten Phase werden zur weiteren Detaillierung bestimmter Themen bzw. Themenbereiche Nachfragen gestellt, die bisher nicht erwähnt wurden, aber aus Sicht der Interviewerin interessant sind. Neuere Entwicklungen in der Biografieforschung verwenden auch weniger streng geregelte Interviewformen und kombinieren narrative Phasen mit thematisch strukturierten oder problemzentrierten Phasen. Meine Interviewführung orientiert sich an diesen Methoden.

$7 \mathrm{Zu}$ Forschungen zur Identitätskonstruktionen in Erzählungen siehe Bamberg (2004, 2006) und Georgakopoulou (2006). In Anlehnung an Antaki/Widdicombe (Hg.) (1998) verstehen die Autoren „Identitäten als dialogisch und relational konstruiert, als in interaktiven, lokalen Prozessen gestaltet und immer wieder umgestaltet" (Keim et al. 2012, S. 33).
} 
Formen unterschieden, zwischen Selbst- und Fremdpositionierungen in der erzählten Zeit und der Relation zwischen erzählendem und erzähltem Ich. Mit der Fremdpositionierung geht meist eine komplementäre Selbstpositionierung einher. Selbst- und Fremdpositionierungen werden sowohl auf der Ebene der Darstellung vergangener Ereignisse als auch auf der Ebene der aktuellen Interaktion im Interview vollzogen. Sie betreffen sowohl das erzählte als auch das erzählende Selbst und können sich aus der Unterschiedlichkeit der diachronen und interaktionalen Perspektive ergeben. Ausgehend von Bambergs Positionierungskonzept (1997) beschreibt Deppermann (2013) folgende Ebenen der Identitätskonstitution:

Auf der ersten Ebene wird die zeitliche und biografische Dimension von Identität verhandelt, das Handeln des Erzählers gegenüber anderen Charakteren in der erzählten Welt. Besondere Anforderungen sind biografische Kontinuität und Übereinstimmung bzw. Distanz zwischen früheren und jetzigen Beurteilungen. Auf der zweiten Ebene werden interaktionsrelevante Aspekte von Identität zwischen dem Erzähler und anderen Interaktionsbeteiligten in der konkreten Erzählsituation verhandelt. Hier spielt das recipient design, d.h. der Adressatenzuschnitt von Äußerungen, eine entscheidende Rolle: Indem Sprechende ihre Beiträge auf Vorannahmen, Wissen und Erwartungen zuschneiden, die sie beim Adressaten aufgrund gemeinsamer Erfahrungen oder der Zugehörigkeit zu bestimmten sozialen Kategorien vermuten, positionieren sie ihn als Person mit bestimmten Eigenschaften und Einstellungen. Auf der dritten Ebene werden Beziehungen und Verknüpfungen zwischen dem lokalen Geschehen und allgemeinen, übergreifenden sozialen Diskursen mit Bezug auf gesellschaftliche Werte und Normen verhandelt.

$\mathrm{Zu}$ den Komponenten des Positionierungskonzepts gehören „Agency“, soziale Kategorisierung und ethnografisches Wissen. Mein besonderes Augenmerk liegt auf der Komponente „Agency“. Mit Referenz auf Lucius-Hoene/Deppermann (2002) ist „Agency“ ein Konzept, in dem linguistisch konstruierte Handlungsspielräume sichtbar werden (ebd., S. 59). Es umfasst das, was der Erzähler macht, in dem er dem Geschehen in seinen Erzählsätzen eine Urheberschaft sprachlich zuordnet. Nach Deppermann (2015 ) können Zuweisungen von „Agency“ sich auf der ganzen Spannbreite zwischen maximaler Handlungsmacht einerseits und Passivität bzw. Widerfahrnis andererseits ansiedeln. Durch linguistische Mittel der Steigerung bzw. der Abschwächung kann der Erzähler die Darstellung von Agentivität modalisieren je nachdem, wie er seine Erlebnisse wahrnimmt; als Zufall, Schicksal oder von höheren Mächten herbeigeführt.

Lucius-Hoene (2012) beschäftigt sich mit der Frage nach dem subjektiven Erleben von Handlungsfähigkeit und Wirkmächtigkeit aus der Perspektive der Erzähler, wie sie dem Geschehen in ihren Erzählsätzen eine Urheberschaft sprachlich zuordnen. Die Autorin plädiert für eine „Agency“-Analyse auf drei Ebenen: auf der Ebene der Erzählung und ihrer sprachlichen Merkmale; auf der Ebene der interaktiven Erzeugung und der involvierten Rollen und auf der Ebene der Geschichtenversion und -moral. Die drei Ebenen überlagern sich oft im Akt des Erzählens und können nur analytisch getrennt werden.

Für die Analyse der sprachlichen Darstellung von Erfahrungen, Erlebnissen und Ereignissen sind die Erzählsätze von Interesse, die zum einen zeigen, dass etwas geschieht, das die Handlung oder die Ereignisfolge der Erzählung vorantreibt, und zum anderen vermitteln, dass eine Person oder etwas dieses Geschehen zustandekommen lässt und daran beteiligt ist. In solchen Sätzen sind die Prädikatsausdrücke und die durch sie bestimmten semantischen Rollen als analytische Instrumentarien von Bedeutung. Prädikatsausdrücke in typi- 
schen Sätzen, die auf Fragen wie ,was passiert dann?“"Auskunft geben, sind nach LuciusHoene (2012, S. 49) zum Beispiel:

- Aktionsprädikate, sie indizieren eine Handlung oder Tätigkeit;

- Prozessprädikate, sie stellen einen Vorgang dar;

- Statusprädikate, sie beschreiben einen Zustand, der veränderlich ist;

- Qualitätsprädikate, sie beziehen sich auf dauernde unveränderliche Eigenschaften.

Prädikatsausdrücke zeigen einerseits, um was für eine Art des Geschehens es sich in der Geschichte handelt, andererseits bestimmen sie die semantischen Rollen, in denen die beteiligten Personen und Wirkmächte auftreten. D.h. wie der Erzähler sich selbst und andere in Bezug auf das Geschehen sieht, wird durch die semantischen Rollen vermittelt, die von den Prädikatsausdrücken zugewiesen werden (vgl. ebd., S. 50).

In Bezug auf prädikatsabhängige semantische Rollen geht es auch um Fragen wie: Wer hat was getan? Wem ist was geschehen? Was hat sich verändert? Diese Rollen können durch Personen, Institutionen, Naturkräfte, soziale Bewegungen etc. ausgefüllt werden. LuciusHoene (ebd., S. 51) führt in Anknüpfung an von Polenz (1988, S. 169-174) folgende semantische Rollen auf, die für die Analyse geringer Handlungsmacht oder Passivität Bedeutung haben:

- Eine Handlung oder Interaktion ist ausgerichtet auf eine Person.

- Eine Person erfährt an sich einen psychischen oder physischen Vorgang oder Zustand.

- Eine Person ist von einer Handlung betroffen.

- Ein Sachverhalt stellt die Ursache für einen anderen Sachverhalt dar, auch als kausale Verknüpfung.

- Eine Person, Sache oder Handlung wird bei einer Handlung vom Agens als Instrument (Werkzeug, Mittel) genutzt.

Mittels dieser Analyseinstrumentarien, den Prädikatsausdrücken und den semantischen Rollen, sind die Agentivitätsanalysen in Bezug auf das Erleben des Erzählers und der in der Geschichte handelnden Personen präzise zu beschreiben. Um die Agentivität der in den autobiografischen Narrationen dargestellten Personen analytisch zu erfassen, folge ich der von Lucius-Hoene (2012, S. 53 f.) angeführten Suchheuristik:

- Wie vollzieht sich das Geschehen, die Handlung oder der Vorgang?

- Wer/was handelt? Wodurch kommt das Geschehen voran? Um welche Art von Wirkmächten handelt es sich?

- Welche semantischen Rollen werden eingeführt?

- Liegt Handlung oder Geschehen als anonymes Ereignis vor? Wird der Handlung Absicht unterstellt?

- Welche semantischen Rollen sind dem Erzählen zugeordnet?

- Wie verhalten sich andere Personen der Geschichte zur Handlung oder dem Geschehen bzw. zum Erzähler?

- Ändern sich die semantischen Rollen der Personen/Instanzen im Verlauf der Geschichte? 


\subsection{Traumakonzept}

In einem Beitrag von 2005 beschäftigen sich Deppermann/Lucius-Hoene mit Verfahren der Darstellung traumatischer Erlebnisse, mit den unter beeinträchtigenden Bedingungen von Krieg, Unfällen, Krankheit und Verlust zustande gekommenen biografischen Erfahrungen. Die Autoren weisen dabei auf eine große Spannbreite an traumabezogenen Darstellungsphänomenen hin. Sie korrespondieren mit den sehr unterschiedlichen moralischen und kognitiv-interpretativen Relevanzen, die Traumata für die Betroffenen haben können, der früheren und heutigen emotionalen Betroffenheit und Bewältigung, der sprachlichen Darstellbarkeit des Erlebten und der Fähigkeit, es deskriptiv und performativ in einem intersubjektiv-diskursiven Feld als traumatisierte Person zu verorten.

Im Mittelpunkt der Untersuchung stehen Erzählstruktur und -dynamik, die sprachlichen und kommunikativen Strategien des Erzählers, und seine prosodischen, stimmlichen und nonverbalen Ausdrucksphänomene. Von besonderem Interesse ist dabei die emotionale Beteiligung, die subjektive Perspektive und Bewertung. Die Darstellbarkeit des Erlebten kann sich ansiedeln zwischen der vollständigen Unfähigkeit, die traumatische Situation zu erzählen, bis zur ausgebauten Erzählung, die zahlreiche Verfahren des Ausdrucks subjektiver Perspektive und Betroffenheit ermöglicht und erfordert.

In dem Gespräch mit Furkan wurden zwei unterschiedliche Typen des Erzählens von Erfahrungen in der Kindheit in Deutschland und in Jugend- und Erwachsenenalter in der Türkei gefunden:

- Die Darstellung von Erfahrungen in Deutschland hat durchgängig den Charakter einer narrativen Verarbeitung von traumatischen Erlebnissen. Im Fokus steht das hilflose Kind, dem unerklärliche Ausgrenzung und Gewalt widerfahren.

- Die sukzessive Annäherung an die erlebte Bedrohung und Ausgrenzung erfolgt über mehrere Erzählepisoden.

- Die Erfahrungen in der Türkei werden im Unterschied dazu eher als Anpassungsprobleme geschildert und Ausgrenzungserfahrungen als Reaktion auf die (noch) nicht geglückte Anpassung dargestellt.

Die Analyse der Gesprächsdaten versucht auch unter Bezug auf die Traumaforschung folgenden Fragen nachzugehen:

- An welchen Stellen im sequenziellen Verlauf des Gesprächs gibt es Phasen, die als traumatische Situation dargestellt werden?

- Welche sprachlich-kommunikativen Mittel werden verwendet?

- Welche Rolle spielen subjektive Beteiligung, „Agency“ und emotionale Betroffenheit?

- Werden moralische Aspekte verhandelt?

Eine Studie, die biografie-, erzähl- und interaktionsanalytisch die Darstellung von traumatisch erlebten ethnischen, sprachlich kulutrellen Ausgrenzungserfahrungen von deutsch-türkischen „Rückkehrern“ zu erfassen versucht, steht noch aus. Hierzu soll mit der vorliegenden Untersuchung ein Beitrag geleistet werden. 


\section{Auswahl des Informanten und Materialbeschreibung}

Das ca. 40-minütige Gespräch mit Furkan findet in meinem Arbeitszimmer an der Marmara-Universität/Istanbul statt. Zum Zeitpunkt des Interviews (2012) ist Furkan 24 Jahre alt und im letzen Studienjahr der Germanistik an der Universität. Die Analyse folgt dem Lauf der biografischen Ereignisse, wie sie Furkan in der narrativen Präsentation, die sich im Wesentlichen an der chronologischen Abfolge orientiert, darstellt. Auffallend ist, dass die Schilderung von Erlebnissen in Deutschland zunächst aus der kindlichen Beobachterperspektive erfolgt, dann zunehmend szenisch detailliert aus der Innenperspektive, und schließlich aus der Erwachsenenperspektive explizit gedeutet im Rahmen von „Rassismus in Deutschland“.

Furkan wuchs in Deutschland in einer Kleinstadt im Süden Baden-Württembergs auf. Gleich zu Beginn des Gesprächs schildert er Ausgrenzungserfahrungen in der Schule durch Lehrpersonen. Als ihm in der fünften Klasse die Versetzung in die Sonderschule droht, entscheidet sich die Familie im Jahr 2000 mitten im Schuljahr in die Türkei auszuwandern. Im türkischen Gymnasium in Istanbul erlebt Furkan, dass er als sprachlich und sozial auffälliger „Halbdeutscher“ abgelehnt wird. Im Anschluss erfolgt der erste Rückblick auf sein Leben in Deutschland, auf Bedrohungs- und Ausgrenzungserlebnisse mit ersten szenischen Details und einer expliziten Deutung im Rahmen von „Rassismus in Deutschland“. Diese retrospektive Deutung wird für ihn und seine Familie erst Jahre später nach der Aufdeckung der NSU-Mordserie möglich. In maximalem Kontrast zu den Erfahrungen in Deutschland stellt Furkan seine Erfahrungen bei einem Auslandsschuljahr in Amerika dar. Hier erlebt er zum ersten Mal das Gefühl von Zugehörigkeit in einer multiethnischen Lebenswelt. Nach der Rückkehr in die Türkei entscheidet er sich für ein Germanistikstudium, und in der „Rückkehrergruppe“ an der Universität gelingt es ihm, ein Gefühl von Zugehörigkeit zu entwickeln. Dort lernt er auch, sich in die türkische Lebenswelt einzupassen. Danach erfolgt ein zweiter Rückblick auf Deutschland mit einer weiteren Detaillierung und Präzisierung von dramatisch erlebten Feindseligkeiten, dargestellt in Kurzszenen. Die retrospektive Deutung dieser einschneidenden Erlebnisse als „rassistisch“ erfolgt wieder über den Hinweis auf die NSU-Morde.

Die Schilderung der aktuellen Lebenssituation zeigt, dass Furkan die traumatischen Erfahrungen in Deutschland überwunden hat. Er ist entschlossen eine erfolgreiche Berufsausbildung abzuschließen.

\section{Leben in Deutschland}

4.1 Der Kindergarten: „hat ich nich viel Probleme“"

Die Interviewerin (ME) leitet das Gespräch mit Furkan (FA) folgendermaßen ein: kannst du über deine erFAHrungen in: ${ }^{\circ} \mathrm{h}$ äh in deutschland, oder in deinem LEbensumfeld erzëHlen; [wie war] s im KINdergarten zum beispiel (006-009), und fragt nach dem Sprachgebrauch in der Familie. Darauf antwortet Furkan folgendermaßen:

017 ME also die sprache WAR deutsch?

018 FA ja [WIR s]äh sprechen öfters DEUTSCH in (.) in unserer familie

[abe ] ${ }^{\circ} \mathrm{h}$ ja manchmal SPRECHT meine (.) äh

$019 \mathbf{M E} \quad[\mathrm{hm}]$

großmutter ts 


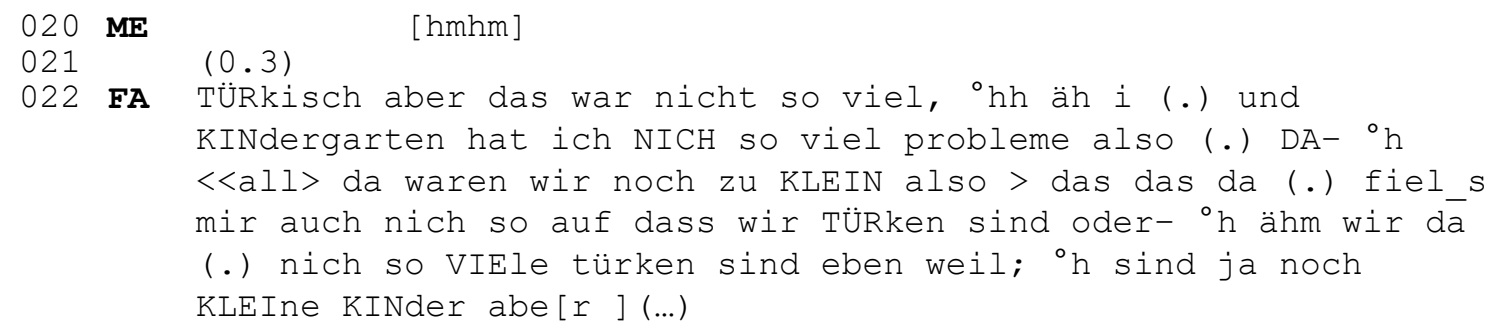

Furkan greift die Vorgabe der Interviewerin auf (also die sprache WAR deutsch? (017)) und bestätigt, dass vor allem Deutsch die Familiensprache war; nur mit der Großmutter hat er Türkisch gesprochen. Anschließend wechselt er selbstinitiiert von der familiären Sprachpraxis zu seinen sozialen Erfahrungen im Kindergarten. Aus meiner bisherigen Erfahrung mit Rückkehrern wäre an dieser Stelle zu erwarten, dass die Darstellung der Kindergartenzeit durchgehend positiv ist. Die meisten Informant/innen erinnern sich an ein breites Spielangebot und an viele Freunde (vgl. auch Keim 2008, S. 133). Im Kontrast dazu schildert Furkan keine positiven Kindergartenerlebnisse, sondern charakterisiert aus der Negation heraus seine damaligen Erfahrungen als weniger problematisch (hat ich $\mathrm{NICH}$ so viel probleme (022)). Diese Formulierung impliziert, dass er in späteren biografischen Phasen ,mehr Probleme' hatte, Probleme, die im Kindergarten für ihn noch nicht erkennbar waren. Dann deutet er die Ursache für seine späteren Probleme an: da (.) fiel_s mir auch nich so auf dass wir TÜRken sind oder $-{ }^{\circ} \mathrm{h}$ ähm wir da (.) nich so VIEle türken sind eben weil; (022). Aus der heutigen Perspektive sieht er als mögliche Ursache für die damaligen Probleme seine ethnische Herkunft. Furkan schafft damit gleich zu Beginn seiner biografischen Darstellung eine Verbindung zwischen seiner türkischen Herkunft und „Probleme haben“ in Deutschland. Mit dieser Rahmung deutet er vorausgreifend die Art von Widrigkeiten an, die er im Folgenden schildert und die er im Zusammenhang mit seiner ethnischen Herkunft interpretiert.

\subsection{Diskriminierungserfahrungen in der Grundschule}

Direkt im Anschluss thematisert Furkan seine Grundschulerfahrungen und wechselt dabei ins Türkische. Das ist auffallend, da er der einzige unter meinen 40 Informant/innen ist, der bei der Schilderung von Erfahrungen in Deutschland ins Türkische wechselt:

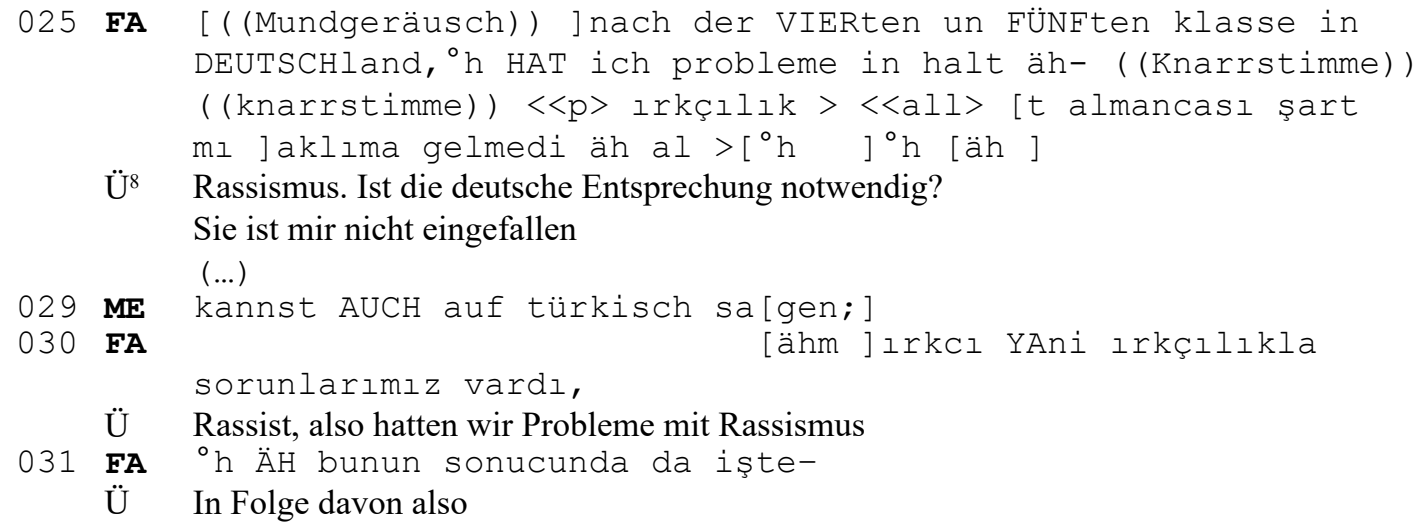

$8 \quad \ddot{U}=$ Übersetzung. 


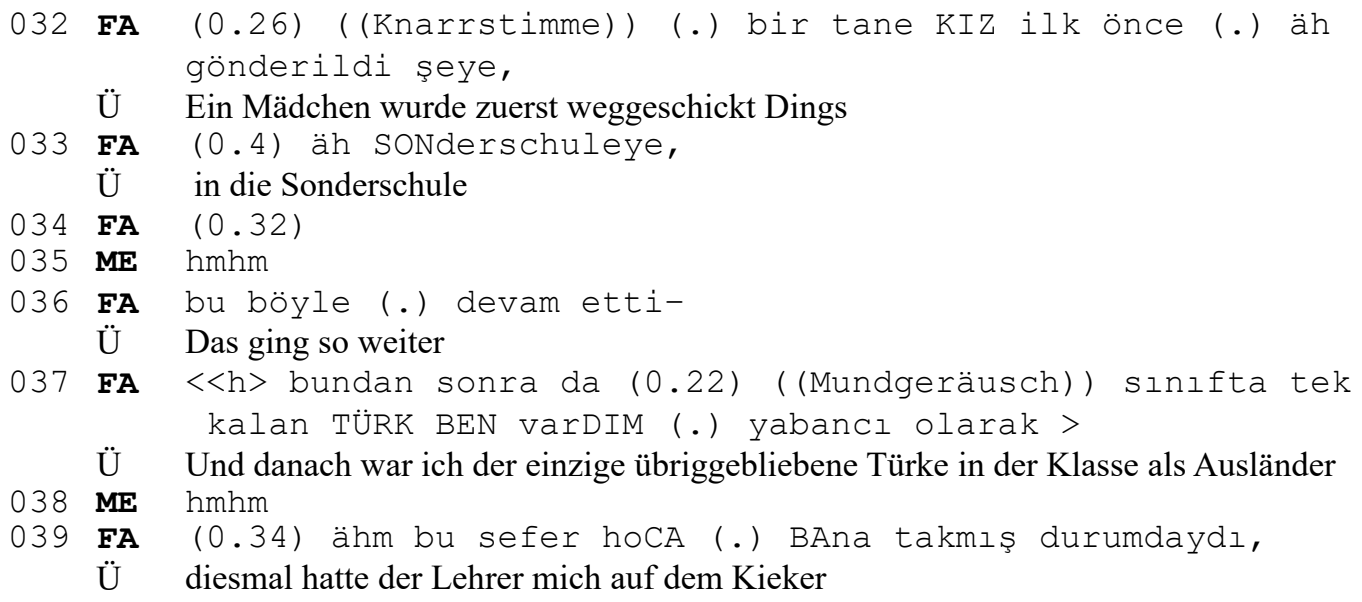

Durch die Angabe nach der VIERten un FÜNFten klasse in DEUTSCHland (025) markiert Furkan den Beginn seiner problematischen Lebensphase: HAT ich problem (025). Da ihm die passende deutsche Bezeichnung für die Charakterisierung der Probleme fehlt, wechselt er ins Türkische: $\langle<p>\imath r k c ̧ ı l ı k>(025)$, Rassismus'. Dann kommentiert er den Sprachwechsel: $\langle<a l l\rangle$ [t almancası şart mı]aklıma gelmedi äh al > (025), Ist die deutsche Entsprechung notwendig? Sie ist mir nicht eingefallen'. Die Interviewerin kommt ihm bei der Sprachwahl entgegen, woraufhin er nochmals das Problem benennt: ırkc (030), Rassist‘. Dass Furkan die deutsche Bezeichnung nicht kennt, lässt vermuten, dass er die Bedeutung von ,Rassismus in Deutschland“ erst nach der Rückkehr in die türkischsprachige Lebenswelt erfahren hat. Mit der nächsten Äußerung schildert er die Probleme seiner Kindergruppe: YAni ırkçılıkla sorunlarımız vardı (030), ,also wir hatten Probleme mit Rassismus'. Die Nennung der Kategorie „Rassist", „Rassismus“, eine auffallende und extrem hochgestufte Rahmung für die Deutung von Erfahrungen der Grundschulkinder zeigt, dass Furkan hier nicht aus der Perspektive des damaligen Kindes spricht, sondern die damaligen Ereignisse in der aktuellen Gesprächssitutation retrospektiv interpretiert.

Solche Prozesse, in denen aus der heutigen Perspektive die Vergangenheit neu gedeutet wird, nennt Brockmeier (1999) retrospektive Teleologie. ${ }^{9}$ Nach diesem Konzept wird eine Lebensgeschichte vom Ende aus neu geschrieben und - in unserem Fall - ihr Verlauf auf das Erleben von Rassismus zugespitzt. In der nächsten Äußerung beschreibt Furkan, wie sich das, was er aus der heutigen Perspektive als „Rassismus“ bezeichnet, für die Kinder damals ausgewirkt hat: KIZ ilk önce (.) äh gönderildi şeye, (0.4) äh sonderschuleye, (032/033) ,Ein Mädchen wurde zuerst weggeschickt Dings in die Sonderschule'.

Zunächst wurde ein Mädchen, das nicht näher spezifiziert wird, aus der Grundschulklasse herausgenommen und in die Sonderschule überwiesen. Danach werden weitere, wiederum nicht spezifizierte Kinder in die Sonderschule überwiesen. Diese Vorgänge beschreibt Furkan aus der Beobachterperspektive des damaligen Kindes: bu böyle (.) devam

9 Im Konzept der „Retrospektiven Teleologie“ macht Brockmeier (1999, S. 36) auf die Umkehrung des Zeiteinflusses aufmerksam:

Da das (vorläufige) Ende der Geschichte bereits gegeben ist, wenn ihr Anfang erzählt wird, stellt es sich im autobiographischen Rückblick nur allzu leicht als Resultat einer von eben jenem Anfang ausund auf das gegenwärtige Ende zugehenden Entwicklung dar. 
etti-(036), Das ging so weiter‘. Dargestellt wird die sukzessive Aussonderung von Kindern aus dem Klassenverband, die sich vor den Augen des beobachtenden Kindes vollzieht, ohne dass es dafür Erklärungen gibt. Erst die nächste Äußerung gibt einen Hinweis auf das Auswahlprinzip: $<<\mathrm{h}>$ bundan sonra da (0.22) ((Mundgeräusch)) sınıfta tek kalan TÜRK BEN varDIM (.) yabancı olarak > (037), Und danach war ich der einzige übriggebliebene Türke in der Klasse als Ausländer‘. Nach Abfolge mehrerer gleichverlaufender Aussonderungsverfahren erkennt das Kind das dahinter liegende Prinzip: Alle Betroffenen haben ein Merkmal gemeinsam: Es sind TÜRK/yabancı (037) (Türke/Ausländer). Da er zu derselben Gruppe gehört, geht er davon aus, dass er als „einzig übriggebliebener Türke“ der erwartbare nächste Kandidat sein wird. In der letzten Äußerung nennt er dann auch den Täter: seinen Klassenlehrer, der ihn jetzt , auf dem Kieker' hat (039).

Die narrative Darstellung zeigt zunächst aus der Sicht des damaligen Kindes das Ausgeliefertsein an ein unerklärliches Aussonderungsverfahren. Das Kind erlebt eine Kette von Ereignissen des Weggeschickt-Werdens gönderildi (032), bei dem sowohl der Täter als auch das Auswahlprinzip undurchschaubar sind. Das gesamte Verfahren erscheint willkürlich. Erst am Ende des Aussonderungsprozesses, als die Wahl auf ihn selbst fällt, wird der Vorgang gedeutet: Das Auswahlprizip ist ethnische Herkunft, die Ausgeschlossenen sind „Ausländer“, „Türken“, und der ausschließende Akteur ist ein Vertreter der Bildungsinstitution. Damit wird das gesamte Verfahren ,rassistisch“ gerahmt und gedeutet. Die von dem Kind erlebte Hilflosigkeit und sein Gefühl des Ausgeliefertseins werden folgendermaßen dargestellt:

- das Aussonderungsverfahren liegt außerhalb der Kontrolle der Betroffenen und außerhalb ihrer Verantwortlichkeit;

- sie erleiden das Verfahren passivisch, kennen die Auswahlkriterien nicht;

- sie erleben ein totales Unterworfensein unter eine Macht, die für sie nicht fassbar ist.

Für die linguistische Analyse von Agentivität sind folgende Erzählsätze von Bedeutung. Dabei liegt der Fokus auf Prädikatsausdrücken und auf den durch sie bestimmten semantischen Rollen:

- Der Erzählsatz YAni ırkçılıkla sorunlarımız vardı, (030), also wir hatten Probleme mit Rassismus'. Die Akteure rassistischer Handlungen werden nicht genannt und „wir“ (= Kinder, Grundschüler) sind die Betroffenen. Die Äußerung hat eine nicht-agentive Bedeutung: Die Kinder wurden mit Rassismusproblemen konfrontiert;

- gönderildi (032), wurde weggeschickt' ist vorgangspassivisch formuliert;

- $<<$ h> bundan sonra da (0.22) ((Mundgeräusch)) sınıfta tek kalan TÜRK BEN varDIM (.) yabancl olarak > (037)/, Und danach war ich der einzige übriggebliebene Türke in der Klasse als Ausländer'; die Äußerung im Sinne von ,ich als Ausländer war übriggeblieben“ ist eine Form des Zustandspassivs. Der Täter, der Furkan aufgrund seines Ausländer- und Türke-Seins aussondern würde, wird nicht genannt.

Im weiteren Verlauf des Gesprächs detailliert Furkan seine Erlebnisse: Er erlebt Gewalt, wird von deutschen Mitschülern geprügelt und erfährt Ungerechtigkeit und Missachtung von seiten der Schule. Wenn er sich gegen die Angreifer wehrt, wird er zusätzlich bestraft. Daraufhin spricht die Mutter in der Schule vor. Das Ergebnis des Eltern-Lehrer-Gesprächs 
stellt Furkan in einer Kurzszene dar, bestehend aus der einleitenden Kategorisierung der Lehrerin und einem Lehrerzitat aus der damaligen Gesprächssituation:

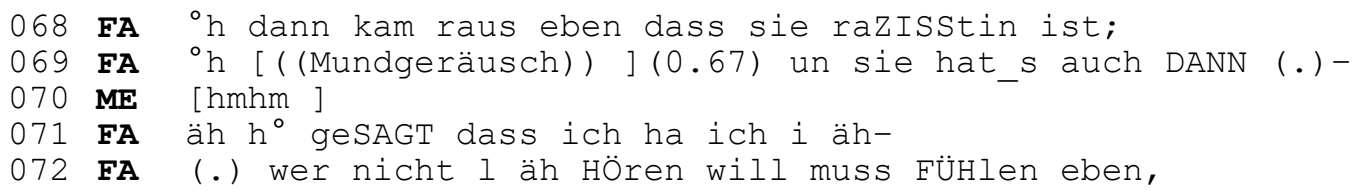

Die Kategorisierung der Lehrerin als Rassistin dann kam raus eben dass sie raZISStin ist; (068) ist das Ergebnis des damaligen Gesprächs aus der Perspektive der Mutter. Im nächsten Schritt führt Furkan ein damaliges Lehrerzitat an: wer nicht 1 äh HÖren will muss FÜHlen eben, (072). Mit dieser Formel lässt er die Lehrerin die erzieherischen Maßnahmen dem Kind gegenüber begründen. Die Formel stammt aus dem Erziehungskontext und fungiert als Begründung für körperliche Strafen beim Fehlverhalten von Kindern und beim Verstoß gegen wichtige Verhaltensregeln. Es ist eine charakteristische Formel aus dem Kontext autoritärer Erziehung, in der auch Gewalt legitimiert wird. Furkan stellt hier einen direkten Bezug zwischen der rassistischen Rahmung und dem autoritäten Erziehungsverhalten her; das impliziert eine Art Automatismus zwischen autoritärer Erziehung und rassistischer Gesinnung.

In der Schilderung zu den Kindheitserlebnissen in Deutschland wird deutlich:

- Es erfolgt eine durchgängige Opferdarstellung des Kindes, sein Ausgeliefertsein an undurchschaubare Ausgrenzungsvorgänge und Gewalterlebnisse;

- auffallend ist, dass ein möglicher Eigenanteil bzw. eine Eigenschuld des damaligen Kindes an den erfahrenen Widrigkeiten nicht in Betracht gezogen wird; z. B. ein auffälliges oder aggressives Verhalten gegenüber Mitschüler/innen und Lehrpersonen, ein mangelnder Lerneifer $u$. ä.

Als Furkan die Versetzung in die Sonderschule ${ }^{10}$ droht, entscheidet sich die Familie für die Rückkehr in die Türkei.

Ein kurzer Blick auf die Bildungssituation ausländischer Kinder Ende der 1990er Jahre in Deutschland, die Zeit, auf die sich Furkans Schulerfahrungen beziehen, ermöglicht es, die geschilderten Erfahrungen in einen größeren gesellschaftlichen Kontext einzuordnen.

\section{Exkurs 1:}

Der Frage, wie und nach welchen Kriterien ausländische Kinder in die Sonderschule selektiert wurden und welche Kenntnisse Eltern über das Auswahl- und Überstellungsverfahren besaßen, ging Uçar ${ }^{11}$ (1996, 2003) nach. Laut der Daten 2001/2002 vom Bun-

10 Sonderschule: allgemeinbildende Pflichtschule für Lernbehinderte, für körperliche oder geistig behinderte oder für schwer erziehbare Kinder und Jugendliche. (Online: www.duden.de/rechtschreibung/Son derschule, Stand: 17.7.2018).

11 Ali Uçar war jahrelang als Grundschul- und Sonderschullehrer tätig, arbeitete als Mitarbeiter im schulpsychologischen Dienst, war Bildungsberater für ausländische Familien und Dozent in der Lehrerfortbildung im Ausländerbereich und an verschiedenen Hochschulen in Berlin. Vor seiner Migration nach Deutschland sammelte er seine Erfahrungen als Lehrer in anatolischen Dörfern und Städten in der Türkei. 
desministerium für Bildung und Forschung besuchten im Jahre 2000 von 950.500 ausländischen Schülern in Deutschland 62.800 Kinder die Sonderschulen. ${ }^{12}$ In den Bundesländern sind die höchsten Ausländeranteile bei Sonderschüler/innen in Hamburg (mit 36,6\%), Baden-Württemberg (mit 34,9\%) und Hessen (mit 31,5\%), die niedrigsten Anteile haben Schleswig-Holstein (mit 9,7\%), Rheinland-Pfalz (mit 16,3\%), Berlin (mit 17,1\%), und Bayern (16,4\%) (ebd., S. 11). Laut dem zehnten Kinder- und Jugendbericht (1998, S. 139) waren etwa zwei Drittel aller Schüler ausländischer Herkunft an Sonderschulen Jungen. ${ }^{13}$

Die Aussonderung der Kinder mit Lernschwäche, Minderbegabung oder Verhaltensstörung ist ein Teil des selektiven deutschen Schulsystems. Leistungsschwache, verhaltensauffällige Kinder werden meistens schon im Laufe der ersten vier Grundschuljahre an die nächst niedere Schulform abgegeben, da Regelschulen oft nicht in der Lage sind sie zu fördern. ${ }^{14}$ Die größte Gruppe bilden Kinder aus benachteiligten sozioökonomischen und soziokulturellen Verhältnissen. Zudem sind ausländische Kinder auch aufgrund ihrer sprachlichen Probleme von dem Auswahlverfahren stärker betroffen als einheimische Kinder. Nach Uçar (1996, S. 13) war die Zahl der ausländischen Kinder, die bis 1989 an Sonderschulen abgegeben wurden, drastisch gestiegen. Powell/Wagner (2001) beschreiben in ihrer Untersuchung, dass nach 1998 ausländische Sonderschüler auch wegen ,,mangelnder Sprachkenntnisse“" und ,körperlicher und motorischer" Auffälligkeiten in Sonderschulen kamen. ${ }^{15}$

Die hohe Anzahl an Versetzungen von Schüler/innen mit Migrationshintergrund in die Sonderschule wirft die Frage auf, ob bei dem Auswahl- und Überstellungsverfahren in die Sonderschule die Eltern der Kinder ausreichend in Kenntnis gesetzt wurden. ${ }^{16}$ Laut einer mündlichen Umfrage (Uçar 2003, S. 12), die türkische Lehrer mit 100 türkischen Probanden (gleichwertig aufgeteilt) in West-Berlin in türkisch durchführten, stellte sich heraus, dass 73,0\% der Eltern keine Kenntnisse über die Sonderschule besaßen. 20\% der Befragten mit geringen Kenntnissen beschrieben die Sonderschule als eine Irrenanstalt, als eine Schule für ausländische Kinder, die nicht Deutsch lernen könnten, oder sie bewerteten die Versetzung in die Sonderschule als eine diskriminierende Maßnahme, in die die ausländerfeindlichen Lehrer die türkischen Kinder abschieben. Von den befragten Eltern gaben nur $11 \%$ an, dass sie dem Zuweisungsverfahren der Sonderschule zugestimmt hatten, während $87 \%$ nicht wussten, ob überhaupt ein solches Verfahren stattgefunden hat. Ebenso hatten $11 \%$ der Eltern einem Testverfahren zugestimmt, ohne zu wissen, dass damit ein

12 Bundesministerium für Bildung und Forschung: Grund- und Strukturdaten 2001/2002, S. 80. In: Uçar (2003, S. 10).

13 Bundesministerium für Familie, Senioren, Frauen und Jugend (BMFSFJ) (Hg.) (1998).

14 PISA und IGLU Studien zeigten, dass im internationalen Vergleich deutsche Schulen damals Kinder und Jugendliche mit Migrationshintergrund benachteiligt behandelten (Keim 2008, S. 94).

15 Bundesministerium für Bildung und Forschung: Grund- und Strukturdaten 1999/2000, S. 70 f. In: Powell/Wagner (2001, S. 14).

16 „Die zuständige Schule hat die Stellungnahme der Erziehungsberechtigten einzuholen, sofern diese nicht selbst den Antrag gestellt haben, und sendet den Antrag mit ihrem Gutachten und dem Vermerk über die Stellungnahme der Erziehungsberechtigenten an das für sie zuständige Schulamt.“ (Nr. 6 Abs. 3 Sonderschulordnung Berlin). 
Schulwechsel verbunden war. 91\% der Eltern wussten von einer endgültigen Entscheidung der Versetzung auf eine Sonderschule nichts.

\section{Leben in der Türkei}

5.1 Die Außenperspektive auf Furkan und die eigene Perspektive auf sich selbst

Nach der Übersiedlung in die Türkei ist der Kampf um soziale und sprachliche Anerkennung groß: Furkan hat schlechte Voraussetzungen für die türkische Schule, er versteht wenig und wird von den anderen als „Halbdeutscher“" bezeichnet.

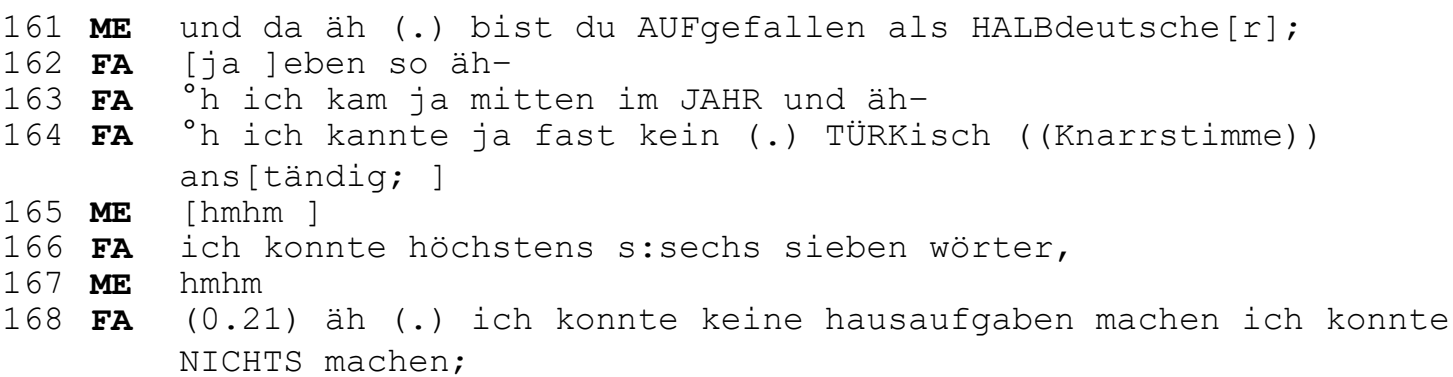

$\mathrm{Zu}$ Beginn übernimmt die Interviewerin die von Furkan vorher verwendete Bezeichnung: HALBdeutsche $[r]$, . Da sie aus eigener Erfahrung weiß, dass einheimische Türken in der Regel für Rückkehrer bestimmte Bezeichungen verwenden, fragt sie an dieser Stelle nicht nach der Bedeutung von „Halbdeutscher“. Später im Gespräch fragt sie: wie hat man dich hier genannt (394) und erhält als Antwort alaMANcı (395), „Deutschländer“17 (vgl. auch Cindark 2010). Das ist neben der deutschen Bezeichnung „Halbdeutscher“ die gebräuchliche türkische Bezeichnung für Rückkehrer/innen. Da Furkan und die Interviewerin das Wissen über die damit implizierte soziale Bedeutung teilen, erfolgt an dieser Stelle keine weitere Erläuterung.

Für die türkischen Mitschüler ist Furkan ein Türke von der Herkunft her, der in Deutschland geboren, aufgewachsen und zur Schule gegangen ist. Er ist ein „Halbdeutscher“, dem die türkische Sprache und Kultur im Wesentlichen fremd sind. Alles, was an ihm als anders oder fremd auffällt, wird als „,deutsch“ interpretiert. Im Anschluss detailliert Furkan, was ihn für seine Mitschüler auffällig machte: Er konnte kaum Türkisch (ich konnte höchstens s:sechs sieben wörter (166)), d.h. er konnte dem Unterricht nicht folgen, es fehlte ihm an schulsprachlichem Vokabular. Außerdem konnte er Texte nicht verstehen oder schreiben (ich konnte keine hausaufgaben machen ich konnte NICHTS machen; 168). D.h. er war unfähig, Aufgaben zu verstehen, Texte zu verfassen, Zusammenhänge zu erklären und zu beschreiben.

17 Cindark erklärt in seiner Arbeit „Migration, Sprache und Rassismus“ (2010, S. 29, Fn. 29):

Die Bezeichnung „Almanc1“ ist eine Kreation der Türkeitürken für die in Deutschland/Europa lebenden Migranten aus der Türkei und wird gewöhnlich mit „Deutschländer“ übersetzt. Das Wort ist eine Synthese aus „Alman“ (Deutscher) und ,Yabanc1“ (Fremder) und könnte demnach auch mit „Fremddeutscher" übersetzt werden. Der Begriff wird von Türkeitürken (oftmals) abfällig gebraucht. 
Auffallend an Furkans narrativer Darstellung seiner Probleme in der Türkei ist, dass er sich einen hohen Eigenanteil zuschreibt. Er nennt seine Defizite in Türkisch, seine Unfähigkeit, dem Unterricht zu folgen und die schulischen Anforderungen zu erfüllen. Die Schilderungen hier stehen in starkem Kontrast zur Schilderung seiner Ausgrenzungserfahrungen in der deutschen Schule, dargestellt als totales Ausgeliefertsein. Jetzt fokussiert er vor allem die eigenen sprachlich-schulischen Defizite, die ihn für die Klassenkameraden , anders' und ,fremd' erscheinen lassen.

Im Anschluss beschreibt er Anpassungsprobleme im sozialen Leben, besonders im kommunikativen Umgang mit anderen. In einer generalisierten Kurzszene stellt er die eigene Perspektive auf eine missglückte Kommunikation mit türkischen Gleichaltrigen dar:

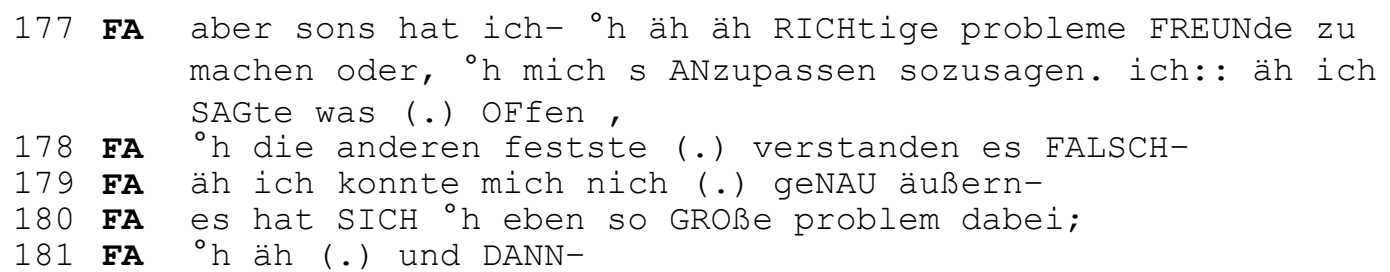

Furkan hatte ,richtige“ Probleme Freundschaften zu schließen und sich an seine Altersgenossen ,anzupassen“. In zwei Zügen von Eigenhandlung und Reaktion der anderen darauf charakterisiert er diese „Probelme“ folgendermaßen: ich: : äh ich SAGte was (.) OFfen , (177), wurde aber von den Gesprächspartnern nur missverstanden. Er expliziert nicht, was „offen“ bedeutet. Aus meiner Erfahrung umfasst „offenes Sprechen“ unabgemildertes, direktes, schnell auf den Punkt kommendes, auch kritisches kommunikatives Handeln. Die Reaktion der Anderen darauf charakterisiert er mit: ${ }^{\circ} \mathrm{h}$ die anderen festste (.) verstanden es FALSCH- (178). Auch hier expliziert er nicht, wie sich Falschverstehen geäußert hat. Aus meiner Erfahrung sind typische Reaktionen auf ,offenes Sprechen“" sich darüber lustig machen, übergehen, abweisen, angreifen, Reaktionen, die Furkan wahrscheinlich ebenfalls erfahren hat. Er bewertet seine damaligen sprachlichkommunikativen Fähigkeiten sehr selbstkritisch: äh ich konnte mich nich (.) geNAU äußern- (179). Ihm fehlte das sprachliche und kulturelle Wissen dazu, wie man sich situativ angemessen ausdrückt.

Die Probleme in der Türkei charakterisiert Furkan durchgehend als Anpassungsprobleme, nicht als Ausgrenzungsprobleme. Er ist der Handelnde, dem das sprachlich-kommunikative Wissen fehlt, um sozial erfolgreich zu agieren. Und er weiß um seine Defizite. Auffällig macht er sich selbst, die anderen reagieren (nur) auf seine mangelnde sozial-kommunikative Kompetenz. Das bedeutet, dass er für seine Ausgrenzungserfahrungen selbst die Verantwortung trägt und es in seiner Macht steht, die Ursachen für die Ausgrenzung zu bekämpfen. Für die Agency-Analyse bedeutet das: Im Gegensatz zu den Darstellungen der Erfahrungen in Deutschland schreibt sich Furkan einen hohen Eigenanteil an Schuld für die misslungene Kommunikation mit gleichaltrigen Türken zu. Freundschaften scheitern an seiner eigenen Unfähigkeit sich sozial und kommunikativ angemessen zu verhalten.

Kurze Zeit später zieht Furkan ein erstes Resümee: Durch die dramatischen Erfahrungen in Deutschland kommt er geschädigt in die Türkei, kann die sozial-kommunikativen Anforderungen dort nicht erfüllen und entscheidet sich für den sozialen Rückzug: hab mich auch etwas zurückgezogen (300). 


\subsection{Erster Rückgriff auf die dramatischen Erfahrungen in Deutschland}

Nach der Schilderung der Erfahrungen in der Türkei leitet die Interviewerin einen Rückblick auf Furkans Erfahrungen in Deutschland ein; sie fragt, ob er auch von Schulkameraden verprügelt worden sei:

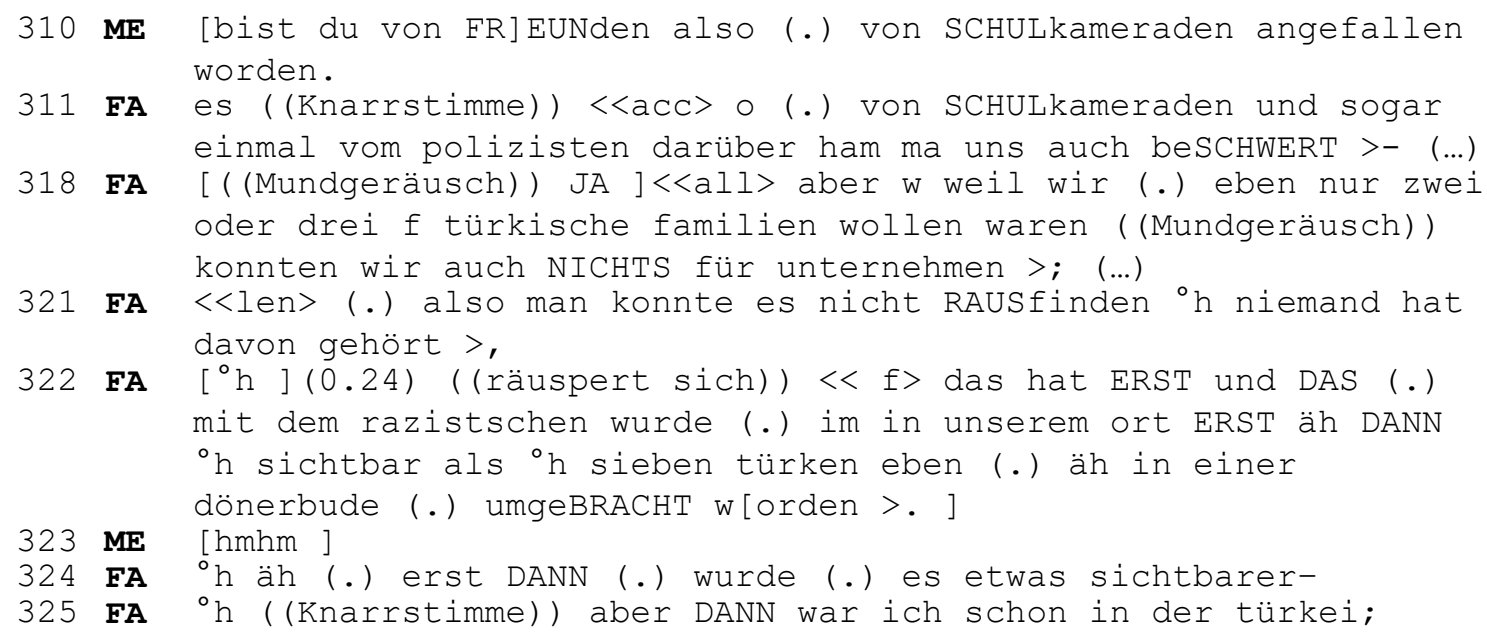

Die Frage der Interviewerin bestätigt Furkan; Schulkameraden haben ihn angegriffen, einmal sogar ein Polizist. Daraufhin beschwerte sich die Familie bei der Behörde. Dass die Beschwerden von der Behörde nicht Ernst genommen wurden, erklärte sich die Familie damals damit, dass sie als türkische Gruppe in der Kleinstadt zu klein und zu unbedeutend waren. Erst viel später, mit der Aufdeckung der NSU-Mordserie, fanden die türkischen Familien im Ort eine Erklärung für die erlebten Anfeindungen. Der Hintergrund für die erfahrenen Feindseligkeiten wurde für sie die rassistische Gesinnung der Täter: $<<\mathrm{f}>$ das hat ERST und DAS (.) mit dem razistschen wurde (.) im in unserem ort ERST äh DANN ${ }^{\circ} \mathrm{h}$ sichtbar als ${ }^{\circ} \mathrm{h}$ sieben türken eben (.) äh in einer dönerbude (.) umgeBRACHT w[orden $>$. ] (322).

In Furkans Darstellung kristallisiert sich das gesamte Ausmaß an selbst erlebten Feindseligkeiten in dem Mord an „sieben Türken in einer Dönerbude“. In seiner Beschreibung überlagern sich die in den deutschen Medien zunächst abschätzig bezeichneten Morde als „Dönermorde“ mit der Vorstellung, dass alle Opfer in einem Döner-Restaurant waren. Dass der Hintergrund für die Morde krimineller Rassismus war, wird erst im Jahre 2011 offenbar. D.h. erst lange, nachdem Furkan in der Türkei lebte, wurde für ihn und seine Familie die Deutung der in der Kindheit erfahrenen Feindseligkeiten in der Schwarzwälder Kleinstadt als ,rassistischer Übergriff“ möglich. Und das erklärt möglicherweise auch, wieso Furkan die Bezeichnung , „rkçılık“" Rassismus“ und ihren Bedeutungskontext nur im Türkischen ausdrücken konnte; er hat die Bezeichnung nur in der türkischen Lebenswelt gehört, lange nachdem er die Ausgrenzungserfahrungen in Deutschland gemacht hatte.

Furkan deutet seine Kindheitserlebnisse aus der Perspektive des jungen Erwachsenen im Kontext der NSU-Morde und konstruiert auch für seine Lebenswelt in Deutschland einen tief verwurzelten Rassismus, der erst allmählich etwas sichtbarer- (324) wurde. Die Morde an den Türken werden zum Deutungsrahmen für sein damaliges Erleben in der gleichaltrigen deutschen Jungengruppe. 


\section{Exkurs 2: Die Morde des NSU in Deutschland}

Nach mehr als fünf Jahren Prozessdauer verurteilte am 11.7.2018 das Oberlandesgericht München Beate Zschäpe, Mitglied der rechtsextremistischen Terrorgruppe „Nationalsozialistischer Untergrund“ (NSU) zu einer lebenslänglicher Gefängnisstrafe. Das Gericht sah Zschäpe als gleichberechtigtes Mitglied eines eingeschworenen Terror-Trios, das sich zusammenschloss, um Menschen ausländischer Herkunft aus rassistischen Gründen zu töten. Zwischen dem Jahr 2000 und 2007 starben acht türkischstämmige und ein griechischstämmiger Kleinunternehmer, außerdem eine Polizistin. Lange Zeit suchte die Polizei die Täter im Umkreis der Opfer, verdächtigte Familien und Freunde der Opfer. Erst durch die Selbstentlarvung von Zschäpe 2011, nachdem sich ihre Freunde Mundlos und Böhnhardt selbst getötet hatten, wurde die Mordserie dem NSU zugerechnet.

Das Verfahren gegen Zschäpe war sehr kompliziert, so Kai Mudra in einem Bericht des Mannheimer Morgen, vom 12.7.2018, 73. Jahrgang Nr. 158: Die Taten liegen lange zurück, es war schwierig Zeugen zu finden, die sich erinnern, es sind Akten geschreddert worden. In demselben Artikel finden sich weitere Stellungnahmen: Martin Geiger zieht nach dem vorläufigen Ende des NSU-Prozesses folgende Bilanz:

Die Aufarbeitung muss weitergehen. Bei der Aufklärung sind zu viele Fragen nach wie vor offen: Wie konnte es geschehen, dass eine Gruppe von Neonazis sieben Jahre lang zehn Morde und zig Raubüberfälle begeht, ohne dass sie gefasst und gestoppt wird? Warum hat der Staat bei der Bekämpfung so versagt?

Beim Münchner NSU-Prozess sind diese Fragen nicht verhandelt und so naturgemäß auch nicht beantwortet worden. Talat Kamran, Leiter des Mannheimer Instituts für Integration und interreligiösen Dialog glaubt, dass die Erkenntnisse aus dem Verfahren nur die Spitze des Eisbergs gewesen seien. Daher verlangt er, dass es vermehrt Präventionsarbeit gegen Rechtsextremismus geben soll: „Wir sollen uns die Frage stellen, wie es in einem so zivilisierten Land wie Deutschland zu so einer Mordserie kommen kann“. Auch für Cem Yalçınkaya, stellvertretender Vorsitzender des Migrationsbeirats Mannheim, bleiben einige Fragen offen. „Was hätte verhindert werden können, wenn man von Anfang an in alle Richtungen ermittelt hätte und nicht die Opfer zu Tätern gemacht hätte? Darüber hinaus hätte man klären können, welche Personen bei Sicherheitsbehörden ihre Funktionen missbraucht haben, indem sie das Umfeld des NSU gewähren ließen." Herbert Diemer von der Bundesanwaltschaft sagte jedoch: „Dass wir dieses Urteil haben, ist ein Erfolg des Rechtsstaates.“ Das Urteil muss noch vom Bundesgerichtshof überprüft werden.

\subsection{Gelungene Einpassung in die türkische Lebenswelt}

Kurz nach dem ersten Rückgriff auf die dramatischen Erfahrungen in Deutschland erfolgt wieder ein Blick auf das Leben nach der Übersiedlung in die Türkei. Als es für Furkan in der Schule nicht besser wird, schickt die Familie ihn für einen einjährigen Schulaufenthalt zu Bekannten nach Amerika. Dort erlebt er - das beschreibt er in maximalem Kontrast zu seinen Erfahrungen in Deutschland - zum ersten Mal Akzeptanz in der Schülergruppe; er fällt in einer ethnisch gemischten Jugendgruppe als Türke nicht auf und fühlt sich angenommen. Nach dem Amerikaaufenthalt entscheidet er sich für ein Germanistikstudium in Istanbul. Er will einen Studienplatz in der Deutschen Abteilung der MarmaraUniversität, um mit Menschen zusammenzusein, die wie er aus deutschland kommen- (414) und ähnliche Probleme hatten wie er (416/420). Furkan folgt hier einer für Rückkehrer/innen charakteristischen Überlegung; er will da studieren, wo auch viele 
Deutsch-Türken studierenen, in Germanistik-Abteilungen. Seine Entscheidung begründet er folgendermaßen:

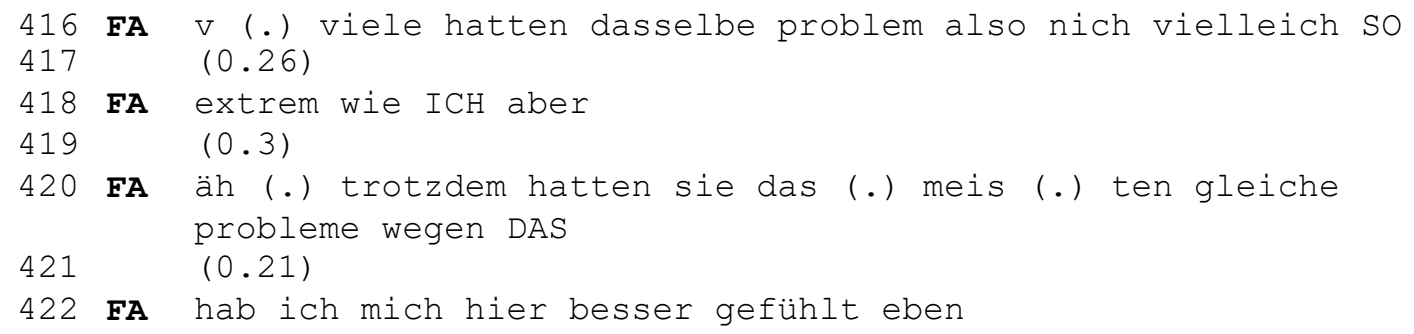

In der Rückkehrergruppe an der Universität gelingt ihm die Einpassung: ich konnte mich BEStens verständigen-(425). Seine neuen Gefährten hätten wie er, wenn auch nicht ganz so schlimm, Erfahrungen mit Ausgrenzung gemacht, sowohl in Deutschland als auch in der Türkei. So werden die neuen Freunde für ihn auch zu einer Art Mentor für das soziale Leben in der Türkei.

Als die Interviewerin nach seinen jetzigen Beziehungen mit einheimischen Studenten fragt, beschreibt er seine aktuelle Situation sehr positiv. Im gesamten Gespräch ist das die einzige Stelle mit agentivisch starken Handlungsdarstellungen. Das macht den folgenden Gesprächsausschnitt zu einer Schlüsselstelle, die zentral ist für den Ausdruck der Bewältigung von Problemen in der Türkei:

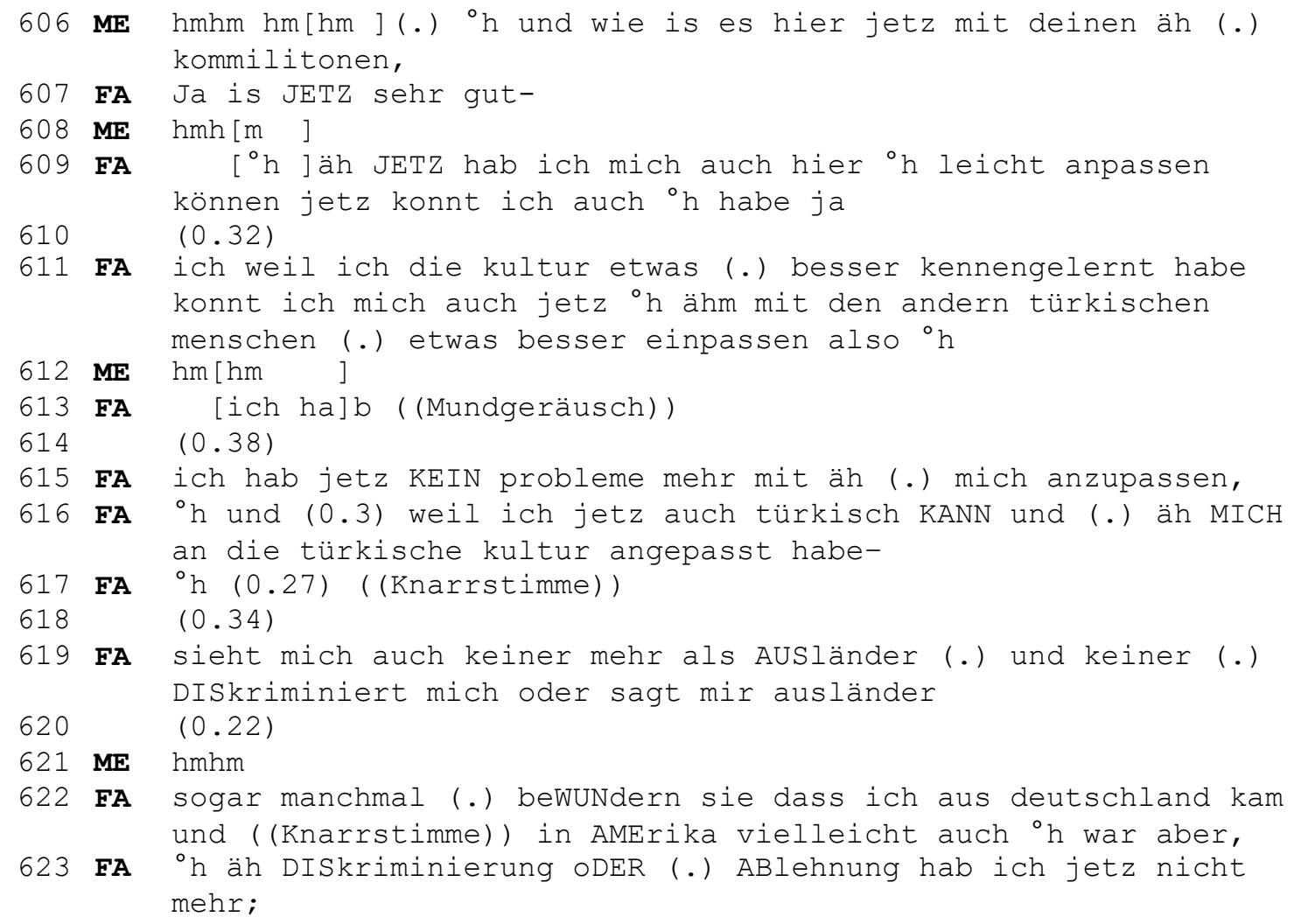

Auf die Frage: und wie is es hier jetz mit deinen g äh (.) kommilitonen, (606) reagiert Furkan mit einer hochgestuften Positivbewertung: ja is JETZ sehr gut- (607). Durch die temporale Deixis ,jetzt“, die er im folgenden sechsmal 
verwendet, hebt er hervor, dass es vor der jetzigen Situation eine Zeit gab, die in starkem Kontrast zu seinem aktuellen Befinden steht. Er führt die Bereiche an, in denen sich die Veränderung festmachen lässt: weil ich die kultur etwas (.) besser kennengelernt habe (611), und es ihm jetzt gelingt, sich ,auch hier anzupassen“, d. h. sich „mit den anderen türkischen Menschen besser einzupassen“ (611). Seinen Erfolg begründet er mit den neu erworbenen Fähigkeiten in Türkisch (weil ich jetz auch türkisch KANN, (616)) und mit dem Erwerb türkischer Kulturpraktiken ( ${ }^{\circ}$ weil ich äh $\mathrm{MICH}$ an die türkische kultur angepasst habe, (616)). D.h., nach vielen Jahren gelingt ihm endlich die Anpassung in seine türkische Lebenswelt. Abschließend verdeutlicht er das Gelingen seiner Anpassung durch die Reaktionen der anderen auf jetziges kommunikatives Handeln. Deren Bewertung, ,jetzt" steht in maximalem Kontrast zu früher:

- sieht mich auch keiner mehr als ausländer (.) und keiner (.) DISkriminiert mich (618). Furkan fällt nicht mehr als „Halbdeutscher“ oder Ausländer auf, er verhält sich sprachlich und situativ angemessen und zeigt, dass er Türke ist;

- sogar manchmal (.) beWUNdern sie dass ich aus deutschland kam und ((Knarrstimme)) in AMErika vielleicht auch ${ }^{\circ} \mathrm{h}$ war, (622). Da ihm die Anpassung gelungen ist, kann er jetzt auch mit Bewunderung für seine „Auslandserfahrung" rechnen. Er wird für den deutschen Anteil in seinem Leben geschätzt, ebenso für seine Erfahrungen in Amerika.

In den Selbstzuschreibungen zeigt Furkan starke Agentivität, die er durch das Personalpronomen ich, die durchgehend aktivischen Formulierungen und durch die mehrfache Verwendung der Deixis jetz ausdrückt, die den Kontrast zu ,früher' hervorhebt. Einige der jetzigen Formulierungen bilden die positiven Versionen zu früheren Negativformaten, wie z.B.:

- ,früher' hatte ich große/richtige Probleme (177) vs. jetzt habe ich keine Probleme,

- ,früher' hatte ich Probleme mich anzupassen (177) vs. jetzt habe ich keine Probleme mich anzupassen (615),

- ,früher' konnte ich fast kein Türkisch (166) vs. jetzt kann ich Türkisch (616),

- ,früher' hat man mich Halbdeutscher genannt (161) vs. jetzt nennt mich keiner mehr Ausländer (619).

\section{Zweiter Rückgriff auf die Diskriminierungserfahrungen in Deutschland}

Im Zusammenhang mit der Schilderung, dass Furkan in beiden Bezugswelten besonders mit gleichaltrigen Jungen Probleme hatte, erfolgt kurze Zeit später der zweite Rückgriff auf die in Deutschland erlebte Ablehnung und Ausgrenzung. Das stellt er in typisierenden, zugespitzten Konfliktszenen dar.

a) Werbung um Anerkennung und Hervorhebung von Gemeinsamkeiten

Die erste Szene besteht aus einem generalisierten Eigenzitat, das Furkan an seine deutschen Angreifer adressiert und in dem er die Gemeinsamkeiten zwischen „sich“ und „ihnen“ hervorhebt: 


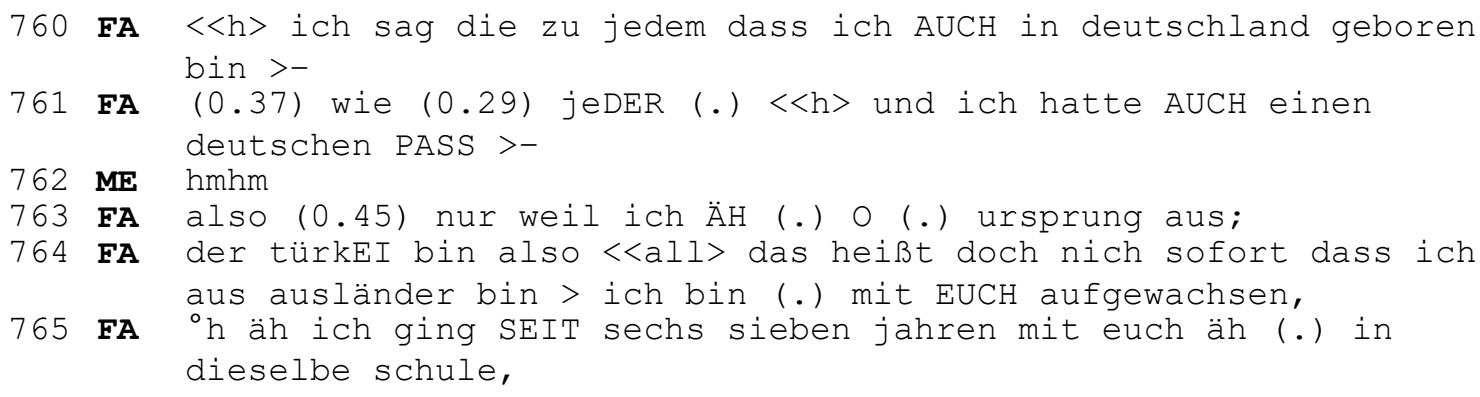

Furkan fordert von seinen Kontrahenten Anerkennung ein. Dazu hebt er eindringlich eine Reihe fundamentaler Gemeinsamkeiten hervor, die er mit ihnen teilt: Sein Geburtsort liegt „auch“ in Deutschland (760), er hat „auch“ den deutschen Pass (761), er ist mit ,ihnen“ aufgewachsen und besucht dieselbe Schule wie sie (764/765). Der einzige Unterschied zwischen ,ihm“ und ,ihnen“ ist die Herkunft seiner Eltern, die aus der Türkei kommen (763/764). Alles, was seine Person betrifft, ist identisch mit den Eigenschaften seiner Kontrahenten; er teilt ihre Lebenswelt und ihre Alltagserfahrungen.

b) Erleben von Gewalt

Danach folgt eine kurze Konfliktszene, bestehend aus einem Eigen- und einem Fremdzitat. Furkan fragt einen Kontrahenten nach den Motiven für seine Feindseligkeit und erhält eine vernichtende Antwort:

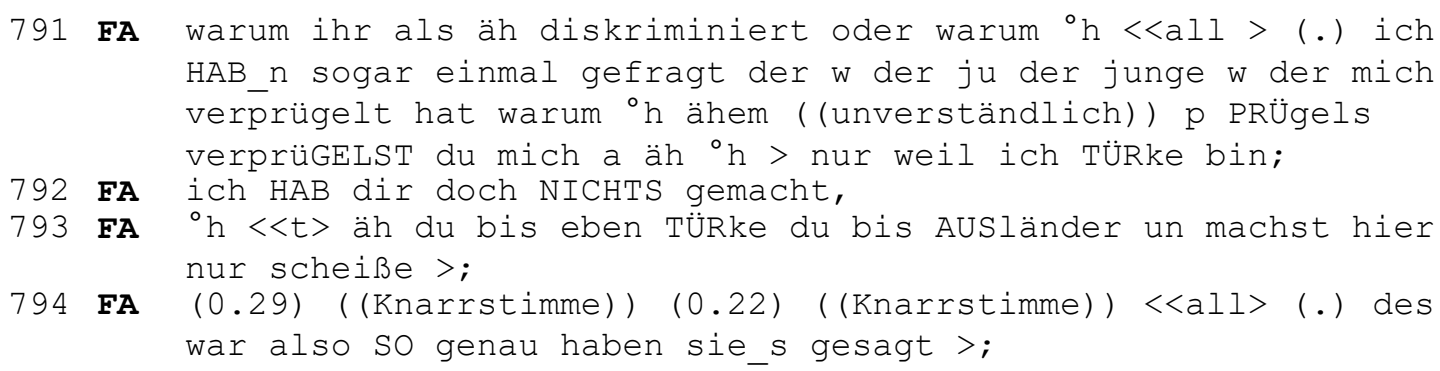

Nach der Frage an den Jungen, warum er prügelt, obwohl Furkan ihm nichts getan hat, führt Furkan selbst ein Motiv für die erlebte Gewalt an: warum ${ }^{\circ} \mathrm{h}$ ähem ( (unverständlich)) p PRÜgels verprüGELST du mich a äh ${ }^{\circ} \mathrm{h}>$ nur weil ich TÜRke bin; (791). D.h. Furkan führt seine ethnische Herkunft als mögliche Begründung für die Feindseligkeiten des Jungen an. Die Selbstverständlichkeit, mit der er die Gewalttätigkeit des anderen ethnisch deutet, zeigt, dass er solche Begegnungen mehrfach erlebte und sich eine Art Routine für solche Konflikbegegnungen herausgebildet hat. Der Junge übernimmt Furkans Begründung und expandiert sie zu einem dreigliedrigen Format: $<<t>$ äh du bis eben TÜRke du bis AUsländer un machst hier nur scheiße >; (793). D.h. der Junge konstatiert, ein, dass es keinen direkten, fallbezogenen Anlass für seine Gewalttätigkeit gibt, sondern dass das Motiv dafür in der Person Furkans begründet ist: in seiner türkischen Herkunft, in seinem Fremdsein und, davon abgeleitet, ganz allgemein in seiner totalen Unfähigkeit und Nutzlosigkeit (machst hier nur scheiße, (793)). Die Szene aus anklagender Frage und vernichtender Zurückweisung, ebenso wie ihre ethnische Deutung haben Routinecharakter. 
c) Erleben ethnisch motivierter Feindseligkeit

Kurz darauf folgt nochmals eine generalisierte Szene, in der Furkan ethnisch motivierte Feindseligkeit unter Jugendlichen beim Fußballspiel erlebt. Die Szene beginnt mit einer kurzen Einführung in die Situation; es spielt eine ethnisch gemischte gegen eine deutsche Mannschaft:

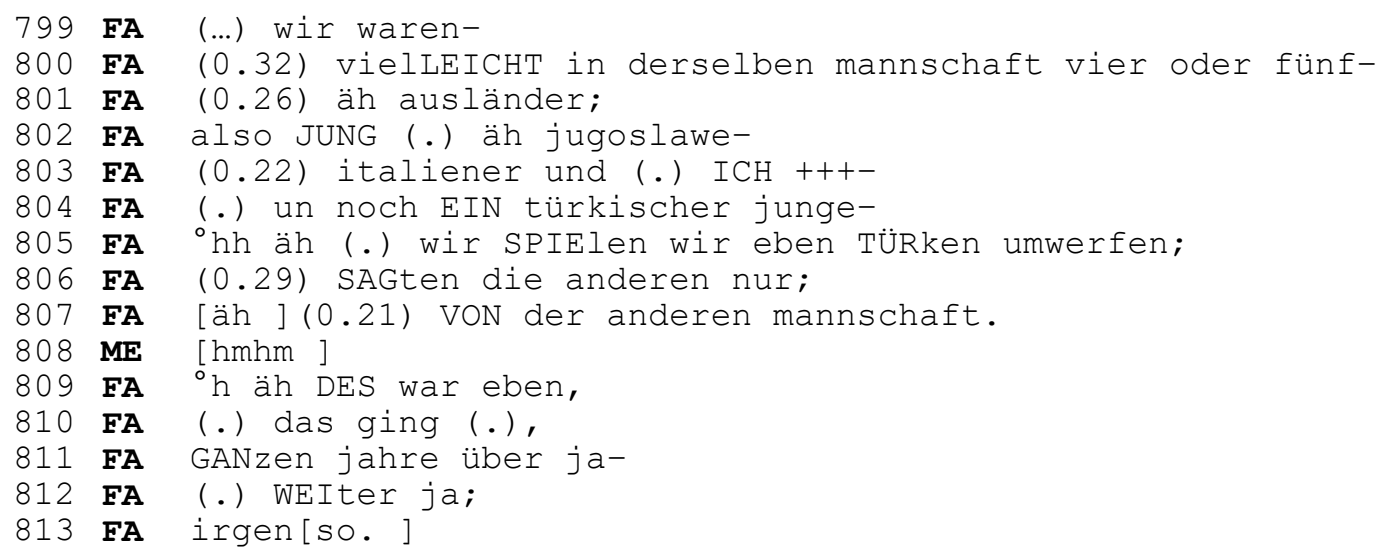

Einleitend wird die gemischte Mannschaft beschrieben; sie besteht aus einem Jugoslawen, einem Italiener und zwei türkischen Jungen $(802,803,804)$. Dann folgt ein Zitat aus der deutschen Mannschaft; ein Spieler schlägt vor: wir SPIElen eben TÜRken umwerfen; (805). Das Zitat ist kein typischer Zuruf beim Fußballspiel, keine Anfeuerung o. ä., sondern eine eindeutig aggressive Aufforderung „Türken umzuwerfen“. Interessant ist, dass aus der Sicht der deutschen Fußballspieler die ethnisch gemischte Gegenmannschaft als „Türken“ bezeichnet wird. Damit bekommt die Zuschreibung Türke - im Vergleich zu Italiener oder Jugoslawe - die Konnotation von besonders ,fremd' und ,feindlich', eine Zuschreibung, die es den Einheimischen erlaubt, dagegen gewalttätig vorzugehen. Der letzte Teil der generalisierten Szene stellt klar, dass das Ereignis keine einmalige Begebenheit war, sondern sich Vorfälle in ähnlicher Weise über Jahre hin ereigneten: GANzen jahre über ja- (.) WEIter ja; irgen[so. ] (811-813).

d) Erklärung für das erlebte Ausmaß an Bedrohung und Gewalt

Dann weitet Furkan die Schilderung von Feindseligkeiten, die er unter Kindern und Jugendlichen erlebte, auch auf die Erwachsenenwelt aus. Die Polizei, die in einem Fall beteiligt war, wies jegliche Verantwortung zurück, und die Zeitung, an die sich die bedrohten türkischen Familien wandten, lehnte eine Veröffentlichung ab. Die türkischen Familien blieben allein.

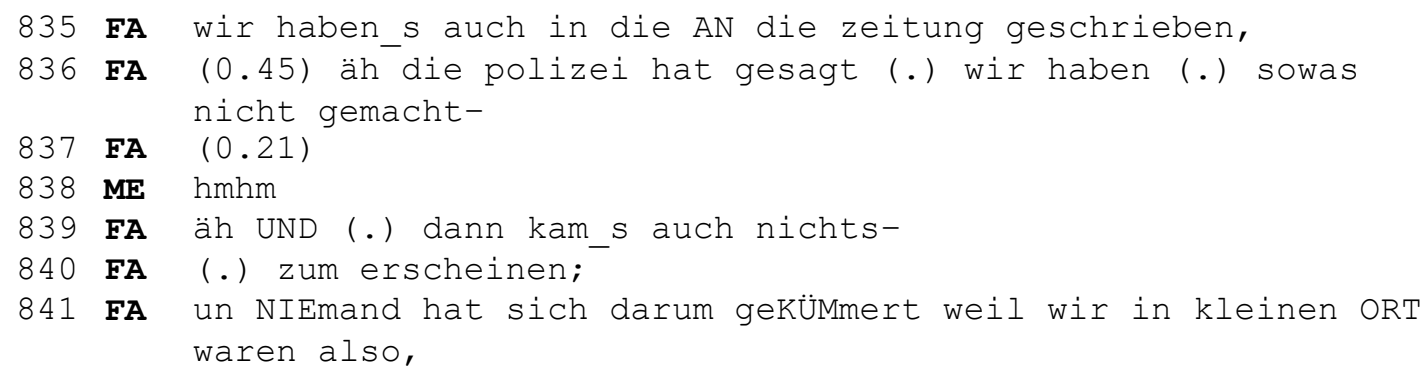




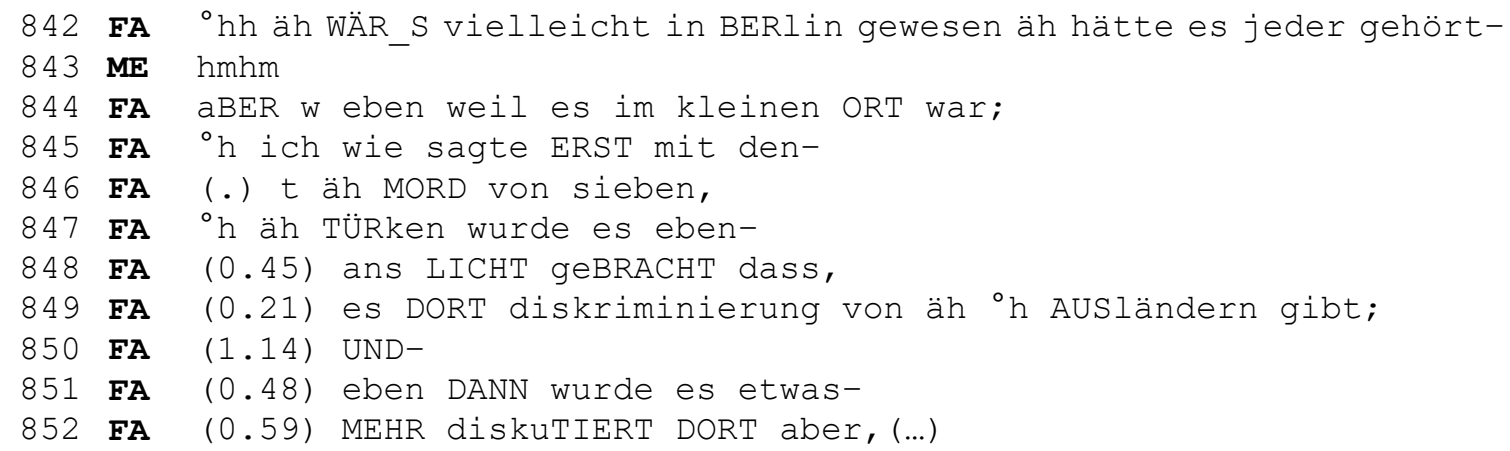

Damals glaubten die türkischen Familien, der Grund dafür, dass ihre Anliegen nirgends berücksichtigt wurden, hänge mit der geringen Größe ihrer Gruppe und auch mit der Abgeschiedenheit ihres Wohnorts zusammen. Es gab niemanden, der sich für ihre Beschwerde interessierte und sich um ihre Anliegen kümmerte. Erst mit der Mordserie des NSU-Trios an Türken wurde für die Familien - so stellt es Furkan dar - die erlebte Feindseligkeit der Deutschen interpretierbar: Die dramatischen Erlebnisse werden retrospektiv als Ausländerfeindlichkeit gedeutet, die es auch in ihrer Schwarzwälder Kleinstadt gab: (...) ERST mit den- (.) t äh MORD von sieben, ${ }^{\circ} \mathrm{h}$ äh TÜRken wurde es eben- (0.45) ans LICHT geBRACHT dass, $(0.21)$ es DORT diskriminierung von äh ${ }^{\circ} \mathrm{h}$ AUSländern gibt; (845-849).

Ein kurzer Ausblick auf Furkans weitere biografische Entwicklung zeigt, dass er die traumatisch erlebten Kindheits- und Jugenderfahrungen überwunden hat. Er schafft es, sein (wie er sagt) „Loser-Image“ zu überwinden. Er absolviert das Germanistikstudium in Istanbul, orientiert sich dann wieder nach Deutschland und schafft die Aufnahme eines Studiums in den Fachbereichen Medienwissenschaft und Linguistik an einer Universität in Deutschland. Dort erreicht er einen überdurchschnittlichen Abschluss. Nach dem Studium arbeitet er eine zeitlang als Journalist beim WDR. Aufgrund der politischen Anspannungen und der bedrohlichen Situation für Journalisten, gibt er den Job wieder auf. Heute ist er als Digitaler Marketing Experte bei einer internationalen Firma in Deutschland tätig.

\section{7. $\quad$ Fazit}

Ein Vergleich der Schilderungen von problematischen Erfahrungen in Deutschland und in der Türkei ermöglicht es, die Erfahrungen in Deutschland unter dem Trauma-Konzept zu fassen. Charakteristisch sind folgende Merkmale:

- Die Aussonderung von Migrantenkindern in der Grundschule: Durch die Undurchschaubarkeit des Aussonderungsprozesses, der auch Furkan droht, erlebt er totale Hilflosigkeit und Ausgeliefertsein an eine höhere Macht.

- Das Erleben von Aggression und Gewalt durch Gleichaltrige: Die Gewaltaktionen entstehen ohne Anlass; Furkan erlebt sie als Bedrohung seiner Existenz.

- Die Angriffe sind initiativ, gelten der gesamten Person. Für den Betroffenen gibt es keine Chance auf eine Änderung der Beziehungen zu den anderen.

- Das Motiv für die Angriffe liegt nicht im Verhalten des Betroffenen, sondern in unveränderlichen Merkmalen seiner Person: seiner Herkunft. 
- Es gibt für ihn keine Möglichkeit, die Angriffe zu stoppen oder ihnen entgegen zu arbeiten.

Im Kontrast dazu werden die Probleme, die Furkan in der Türkei schildert, nicht als traumatische Erfahrungen, sondern als z. T. schmerzhafte Anpassungsschwierigkeiten an eine neue Sozialwelt geschildert. Folgende Merkmale sind charakteristisch:

- Furkan erkennt die eigenen Defizite, durch die er auffällig wird.

- Er erkennt, dass es in seiner Macht und Verantwortung liegt, die Ursachen für die Ausgrenzung abzubauen.

- Die Ausgrenzungsaktivitäten der anderen sind reaktiv, sie erfolgen auf Furkans auffälliges Verhalten.

- Der Grund für die Ausgrenzung liegt nicht in unveränderlichen Eigenschaften seiner Person (Herkunft, Hautfarbe, Religion etc.), sondern in seinem inadäquaten Handeln.

- Sobald es ihm gelingt, sein Verhalten zu ändern und sich den anderen anzupassen, erfährt er Achtung und Bewunderung.

Im Kontrast zu den Schilderungen problematischer Erfahrungen in Deutschland und in den Anfangsjahren in der Türkei ist Furkans Schilderung seiner jetztigen Lebenssituation und seine positive Selbstsicht folgendermaßen charakterisiert:

Die Selbstzuschreibungen sind agentivisch stark formuliert.

- Viele Äußerungen bestehen aus positiven Versionen früherer Negativformate, z.B.: „,ich kann jetzt X“" oder ,ich habe jetzt keine Probleme mehr“.

- Auf der Inhalts- und der Formulierungsebene wird ein maximaler Kontrast zu früheren Darstellungsformen hergestellt.

\section{Anhang: Transkriptionskonventionen}

GAT2 (Selting et al. 2009)

$\underline{\text { Sequenzielle Struktur/Verlaufsstruktur }}$

[ ] Überlappungen und Simultansprechen

[ ]

unmittelbarer Anschluss neuer Sprecher/innenbeiträge oder Segmente (latching)

Ein- und Ausatmen

${ }^{\circ} \mathrm{h} / \mathrm{h}^{\circ}$

${ }^{\circ} \mathrm{hh} / \mathrm{hh}^{\circ}$

${ }^{\circ} \mathrm{hhh} / \mathrm{hhh}^{\circ}$

\section{$\underline{\text { Pausen }}$}

(.)

(-)

$(--)$

$(---)$

$(0.5)$

(2.0)
Ein- bzw. Ausatmen von ca. 0.2-0.5 Sek. Dauer

Ein- bzw. Ausatmen von ca. 0.5-0.8 Sek. Dauer

Ein- bzw. Ausatmen von ca. 0.8-1.0 Sek. Dauer

Mikropause, geschätzt, bis ca. 0.2 Sek. Dauer

kurze geschätzte Pause von ca. 0.2-0.5 Sek. Dauer

mittlere geschätzte Pause v. ca. 0.5-0.8 sek. Dauer

längere geschätzte Pause v. ca. 0.8-1.0 sek. Dauer gemessene Pausen v. ca. 0.5 bzw. 2.0 Sek. Dauer (Angabe mit einer Stelle hinter dem Punkt) 


\section{Sonstige segmentale Konventionen}

und äh

äh öh äm

Verschleifungen innerhalb von Einheiten

Verzögerungssignale, sog. ,gefüllte Pausen“

$::$

$:::$

$?$

Dehnung, Längung, um ca. 0.5-0.8 Sek.

Dehnung, Längung, um ca. 0.8-1.0 Sek.

Abbruch durch Glottalverschluss

\section{Akzentuierung}

akZENT

Fokusakzent

ak!ZENT!

extra starker Akzent

Tonhöhenbewegung am Ende von Intonationsphrase

$\begin{array}{ll}? & \text { hoch steigend } \\ , & \text { mittel steigend } \\ - & \text { gleichbleibend } \\ ; & \text { mittel fallend } \\ \text {. } & \text { tief fallend }\end{array}$

Verändertes Tonhöhenregister

$<<\mathrm{t}>$ was $>$

tiefes Tonhöhenregister

$<<\mathrm{h}>$ was $>$

hohes Tonhöhenregister

Sonstige Konvention

$<<$ erstaunt $>$ was $>$

interpretierende Kommentare mit Reichweite

Lachen und Weinen

ha ha he he hi hi

((lacht))

silbisches Lachen

$<<$ lachend $>$ was $>$

Beschreibung des Lachens

$<<:-)>$ soo $>$

Lachpartikel in der Rede, mit Reichweite

„smile voice“

\section{Rezeptionssignale}

$\mathrm{hm}$ ja nein nee

$\mathrm{hm} \_\mathrm{hm}$ ja_a

nei_ein nee_e

Phm?hm

einsilbige Signale

zweisilbige Signale

mit Glottalverschlüssen, meistens verneinend

\section{Sonstige Konventionen}

((hustet))

$<<$ hustend $>$ was $>$

para- und außersprachliche Handlungen $u$. Ereignisse

sprachbegleitende para- und außersprachliche Handlungen u. Ereignisse mit

Reichweite

unverständliche Passage ohne weitere Angaben

$(\mathrm{xxx}),(\mathrm{xxx} \mathrm{xxx})$

(solche)

ein bzw. zwei unverständliche Silben

vermuteter Wortlaut

(also/alo)

(solche/welche)

mögliche Alternativen

((unverständlich, ca. 3 Sek)) unverständliche Passage mit Angabe der Dauer

$((\ldots))$

Auslassung im Transkript

Lautstärke- und Sprechgeschwindigkeitsveränderungen, mit Extensionen

$<<\mathrm{f}>$ was $>$

forte, laut

$<<\mathrm{ff}>$ was $>$

fortissimo, sehr laut 


$\begin{array}{ll}<<\text { p }>\text { was }> & \text { piano, leise } \\ <<\text { pp }>\text { was }> & \text { pianissimo, sehr leise } \\ <<\text { all }>\text { was }> & \text { allegro, schnell } \\ <<\text { len }>\text { was }> & \text { lento, langsam } \\ <<\text { cresc }>\text { was }> & \text { crescendo, lauter werdend } \\ <<\text { dim }>\text { was }> & \text { diminuendo, leiser werdend } \\ <<\text { acc }>\text { was }> & \text { accelerando, schneller werdend } \\ <<\text { rall }>\text { was }> & \text { rallentando, langsamer werdend }\end{array}$

Veränderung der Stimmqualität und Artikulationsweise

$<<$ creaky $>$ was $>\quad$ glotalisiert, „Knarrstimme“

$<<$ flüsternd $>$ was $>\quad$ Beispiel für Veränderung der Stimmqualität, wie angegeben

\section{Literatur}

Antaki, Charles/Widdicombe, Sue (Hg.) (1998): Identities in talk. London/New Delhi: Sage.

Bamberg, Michael (1997): Positioning between structure and performance. In: Journal of Narrative and Life History, 7, 1-4. S. 335-342.

Bamberg, Michael (2004): Narrative discourse and identitieS. In: Meister, Jan-Christoph et al. (Hg.): Narratology beyond literary criticism. Berlin/New York: De Guyter. S. 213-237.

Bamberg, Michael (2006): Biographic-narrative research, quo vadis? A critical review of „,big stories“ from the perspective of „small stories“. In: Milnes, Kate et al. (Hg.): Narrative, memory and knowledge. Repräsentation, aesthetics and contextS. Huddersfield: University of Huddersfield Press. S. 1-17.

Brockmeier, Jens (1999): Erinnerung, Identität und autobiographischer Prozeß. In: Journal für Psychologie, 7, 1. S. 22-42. (Online: http://nbn-resolving.de/urn:nbn:de:0168-ssoar-28767, Stand: 25.7.2018).

Bundesministerium für Familie, Senioren, Frauen und Jugend (Hg.) (1998): Zehnter Kinder- und Jugendbericht. Bericht über die Lebenssituation von Kindern und die Leistungen der Kinderhilfen in Deutschland. Bonn: BMFSFJ.

Cindark, Ibrahim (2010): Migration, Sprache und RassismuS. Der kommunikative Sozialstil der Mannheimer „Unmündigen“ als Fallstudie für die „emanzipatorischen Migranten“. Tübingen: Narr. (= Studien zur deutschen Sprache 51).

Daimagüler, Mehmet G. (2011): Kein schönes Land in dieser Zeit. Das Märchen von der gescheiterten Integration. Gütersloh: Gütersloher Verlagshaus.

Deppermann, Arnulf (2013): How to get a grip on identities-in-interaction. (What) Does ,Positioning' offer more than ,Membership Categorization“? Evidence from a mock story. In: Bamberg, Michael (Hg.): Narrative inquiry. A forum for theoretical, empirical, and methodological work on narrative, $23,1$. Amsterdam: Benjamins. S. 62-88.

Deppermann, Arnulf (2015): Agency in Erzählungen über Gewalterfahrungen in Kindheit und Jugend. Sprachliche Praktiken der Zuschreibung von Schuld und Verantwortung als Täter und Opfer. In: Scheidt, Carl Eduard et al. (Hg.): Narrative Bewältigung von Trauma und Verlust. Stuttgart: Schattauer. S. 64-75.

Deppermann, Arnulf/Lucius-Hoene, Gabriele (2005): Trauma erzählen - kommunikative, sprachliche und stimmliche Verfahren der Darstellung traumatischer Erebnisse. In: Psychotherapie und Sozialwissenschaft, 7, 1. S. 35-73.

Devran, Serap (2017): Deutsch-türkische Migration. Die Darstellung narrativer Identitäten von Studentinnen in Istanbul. Eine biographie- und interaktionsanalytische Pilotstudie. Mannheim: Institut für Deutsche Sprache. (= amades 51).

Georgakopoulou, Alexandra (2006): Thinking big with small stories in narrative and identity analysis. In: Narrative Inquiry 16, 1. S. 122-130. 
Keim, Inken (2008): Die „türkischen Powergirls“. Lebenswelt und kommunikativer Stil einer Migrantinnengruppe in Mannheim. 2., durchges. Aufl. (= Studien zur deutschen Sprache 39). Tübingen: Narr.

Keim, Inken et al. (2012): Heirat und Migration aus der Türkei. Biographische Erzählungen junger Frauen. (= Studien zur deutschen Sprache 58). Tübingen: Narr.

Lucius-Hoene, Gabriele (2012): „Und dann haben wir's operiert“. Ebenen der Textanalyse narrativer Agency-Konstruktionen. In: Bethmann, Stephanie et al. (Hg.): Agency. Qualitative Rekonstruktionen und gesellschaftstheoretische Bezüge von Handlungsmächtigkeit. Weinheim: Beltz Juventa. S. 40-70.

Lucius-Hoene, Gabriele/Deppermann, Arnulf (2002): Rekonstruktion narrativer Identität. Ein Arbeitsbuch zur Analyse narrativer InterviewS. Opladen: Leske + Budrich.

Lucius-Hoene, Gabriele/Deppermann, Arnulf (2004): Narrative Identität und Positionierung. In: Gesprächsforschung. Online-Zeitschrift zur verbalen Interaktion, 5. S. 166-183. (Online: www.gespraechsfor schung-ozs.de, Stand: 22.7.2018).

Polenz, Peter von (1988): Deutsche Satzsemantik: Grundbegriffe des Zwischen-den-Zeilen-Lesens. Berlin: De Gruyter.

Powell, Justin/Wagner, Sandra (2001): Daten und Fakten zu Migrantenjugendlichen an Sonderschulen in der Bundesrepublik Deutschland. Berlin: Max-Planck-Institut für Bildungsforschung.

Rosenthal, Gabriele (1995): Erlebte und erzählte Lebensgeschichte. Frankfurt a. M.: Campus.

Schütze, Fritz (1983): Biographieforschung und narratives Interview. In: Neue Praxis 3. S. 283-293.

Selting, Margret et al. (2009): Gesprächsanalytisches Transkriptionssystem 2 (GAT2). In: Gesprächsforschung. Online Zeitschrift zur verbalen Interaktion, 10. S. 353-402. (Online: www.gespraechsforschungozs.de/fileadmin/dateien/heft2009/px-gat2.pdf, Stand: 20.7.2018).

Uçar, Ali (2003): Einführung. Migration und Behinderung - zur Sonderschulproblematik von Kindern mit Migrationshintergrung. In: Sonderpädagogik oder Pädagogik der Vielfalt? Dokumentation der Fachtagung in Hannover am 29.9.2003.

Uçar, Ali (1996): Benachteiligt. Ausländische Kinder in der deutschen Sonderschule. Eine empirische Untersuchung zur Lage der türkischen Kinder in der Schule für Lernbehinderte. Baltmannsweiler: Schneider-Verl. Hohengehren.

Dr. Serap Devran

Marmara Üniversitesi

Fen-Edebiyat Fakültesi

Alman Dili ve Edebiyatı Bölümü

Göztepe Kampüsü

34722 Kadıköy/Istanbul

Türkei

E-Mail: sdevran@marmara.edu.tr 